\title{
A Parametric Analysis of the Effect of Hybrid Nanoparticles on the Flow Field and Homogeneous-Heterogeneous Reaction between Squeezing Plates
}

\author{
Wajid Ullah Jan, ${ }^{1}$ Muhammad Farooq, ${ }^{1}$ Aamir Khan, ${ }^{2}$ Asma Alharbi $\left(\mathbb{D},{ }^{3}\right.$ Rehan Ali Shah, ${ }^{4}$ \\ Rashid Jan $\unrhd^{5},{ }^{5}$ and Sahar Ahmed Idris $\oplus^{6,7}$ \\ ${ }^{1}$ Department of Mathematics, Abdul Wali Khan University Mardan, Mardan, 23200 KP, Pakistan \\ ${ }^{2}$ Department of Pure and Applied Mathematics, The University of Haripur, Haripur, KP, Pakistan \\ ${ }^{3}$ Department of Mathematics, College of Science and Arts, Qassim University, Ar Rass, Saudi Arabia \\ ${ }^{4}$ Department of Basic Sciences and Islamiat, University of Engineering and Technology, Peshawar, KP, Pakistan \\ ${ }^{5}$ Department of Mathematics, University of Swabi, Swabi 23561, KP, Pakistan \\ ${ }^{6}$ College of Industrial Engineering, King Khalid University, Abha 62529, Saudi Arabia \\ ${ }^{7}$ Department of Mathematics, College of Sciences, University of Juba, Juba, South Sudan
}

Correspondence should be addressed to Sahar Ahmed Idris; sa6044690@gmail.com

Received 21 October 2021; Accepted 13 January 2022; Published 9 February 2022

Academic Editor: David Carf

Copyright (c) 2022 Wajid Ullah Jan et al. This is an open access article distributed under the Creative Commons Attribution License, which permits unrestricted use, distribution, and reproduction in any medium, provided the original work is properly cited.

\begin{abstract}
Different strategies have been utilized by investigators with the intention of upgrading the thermal characteristics of ordinary liquids like water and kerosene oil. The focus is currently on hybrid nanomaterials since they are more efficient than nanofluids, so as to increase the thermal conductivity of fluids and mixtures. In a similar manner, this investigation is performed with the aim of breaking down the consistent mixed convection flow close to a two-dimensional unstable flow between two squeezing plates with homogeneous and heterogeneous reaction in the presence of hybrid nanoparticles of the porous medium. A sustainable suspension in the ethylene glycol with water is set by dissolving inorganic substances, iron oxide $\left(\mathrm{Fe}_{3} \mathrm{O}_{4}\right)$ and cobalt $(\mathrm{Co})$, to form $\mathrm{Fe}_{3} \mathrm{O}_{4}-$ $\mathrm{Co} / \mathrm{C}_{2} \mathrm{H}_{6} \mathrm{O}_{2}-\mathrm{H}_{2} \mathrm{O}$ hybrid nanofluid. The numerical and analytical model portraying the fluid flow has been planned, and similitude conditions have been determined with the assistance of the same transformations. The shooting technique has been used to solve nonlinear numerical solution. To check the validity of the results obtained from the shooting mode, the Matlab built-in function BVP4c and Mathematica built-in function homotopy analysis method (HAM) are used. The influence of rising parameters on velocity, temperature, skin friction factor, Nusselt number, and Sherwood number is evaluated with the help of graphs and tables. It has been found in this work that to acquire a productive thermal framework, the hybrid nanoparticles should be considered instead of a single sort of nanoparticles. In addition, the velocities of both the hybrid nanofluids and simple nanofluids are upgraded by the mixed convection boundary, whereas they are decreased by the porosity. An augmentation in volumetric fraction of nanoparticles correlates to an increment in the heat transmission rate. It is also found that heat transfer rate for $\mathrm{Fe}_{3} \mathrm{O}_{4}-\mathrm{Co} / \mathrm{C}_{2} \mathrm{H}_{6} \mathrm{O}_{2}-\mathrm{H}_{2} \mathrm{O}$ hybrid nanofluids (HNF) is better than that of the $\mathrm{Fe}_{3} \mathrm{O}_{4}-\mathrm{C}_{2} \mathrm{H}_{6} \mathrm{O}_{2}-\mathrm{H}_{2} \mathrm{O}$ of single nanofluids (SNF). This research shows that hybrid nanofluids play a significant part in the transfer of heat and in the distribution of nanofluids at higher temperatures.
\end{abstract}

\section{Introduction}

Pressure flows have many engineering, scientific, and technical applications in the industry such as lubrication system, moveable pistons, hydrodynamic engines, hydraulic lifts, scattering and formulation, chemical equipment processing, food processing, film damage, and frost damage syringes and nasogastric tubes. The initial study of squeezing flow was published by Stefan [1] who reported the lubrication method in his research. New doors were opened by 
Stephen's rewarding work for researchers on squeezing flows. Different researchers studied the flow of compression and followed him. From different research perspectives, this study has been pushed forward in recent years to compress the flow. Hayat et al. [2] have examined the squeezed flow of MHD fluid between two horizontal disks using homotopy analysis. The result of their investigation was found to be an increase in the velocity field of for augmenting values of micropolar parameter. Mustafa et al. [3] analyzed the fluid flow with magnetic effects upon thermal and mass transmission behavior of an incompressible viscous Casson fluid flow amid parallel plates. They have noticed in this work that flow has been augmented with escalating values of squeezing parameter. The two-dimensional magnetized laminar constant Marangoni convection of the incompressible viscous fluid was explored by Mahanthesh et al. [4] by implementing the Runge-Kutta-Fehlberg technique. The authors have found that the boundary layer thickness and the increasing meridian convection have increased the fluid velocity within the flow area.

Ferroliquids are magnetic nanofluids suspended in nondirecting liquids such as water, hydrocarbons, and kerosene. These ferrofluids have various applications in medical science like cell partition, focusing of drug medication, and imaging of magnetic characters. The thermal and magnet functions of the ferrofluid flow were investigated by Neuringer [5]. Khan et al. [6] examined the influence of a homogeneous heat stream on flat-surface slip flows with heat transfer. They evaluated three distinct ferrofluids with two distinct basic fluids $\left(\mathrm{CoFe}_{2} \mathrm{O}_{4}, \mathrm{Fe}_{3} \mathrm{O}_{4}\right.$, and $\left.\mathrm{Mn}-\mathrm{ZnFe}_{2} \mathrm{O}_{4}\right)$ (water and kerosene). Rashad [7] examined the magnetic slip-flow function containing nonisothermal convection and radiation wedge kerosene based cobalt ferrofluid. Zaib et al. [8] investigated a mixed convective flow entropy of a vertical plate of magnetite ferrofluid. Ali et al. [9] recently discussed the magnetic dipole impact on micropolar fluid consisting of the EG and the water-based ferrofluids $\mathrm{Fe}$ and $\mathrm{Fe}_{3} \mathrm{O}_{4}$ from a stretched sheet.

Hybrid nanoliquids, however, are deliberately captured by blending several different nanoparticles with better thermal and rheological characteristics. The introduction of hybrid ferroliquids is to increase heat transfer efficiency in fluid flow. It has several scientific applications such as dynamic sealing, naval sealing, dampening, and microfluidics. Suresh et al. [10] investigated the effect of dissipation on time-based flux comprising a rounded pipe of hybrid nanoliquid. They have achieved a lower friction factor for nanolytes than for hybrid nanol. The pressurization decline in the volume percentage of the water-based $\mathrm{Cu}-\mathrm{TiO}_{2}$ hybrid nanolic was examined by Madhesh and Kalaiselvam [11]. Minea [12] revealed the association with the date of the temperature gradient of alumina hybrids and nanofluids. The fluid flow characteristics of hybrid nanoliquids from water-based $\mathrm{Ag}-\mathrm{CuO}$ were evaluated by Hayat and Nadeem [13]. Mebarek-Oudina [14] examined the thermal and hydrodynamic parameters of Titania nanoliquids that satisfy a cylinder annulus, the impact of annulus, Mahanthesh et al. [15] An exponential spatially dependent magneto slip heat source flow from an extendable rotation consisting of carbon nanofluids. Marzougui et al. [16] investigated the surface effects. $\mathrm{Al}_{2} \mathrm{O}_{3}-\mathrm{CuO}$ stability roughness and radiation nanolic hybrid through the widespread use of the model. Recently, Wakif et al. [17] examined the entropy examination through convective flow including nanoliquid by means of MHD with chamfers in a hole. The investigation of the attractive capacity has significant sales in MHD orientation; topography, astronomy, siphons, generators, medication, control of limit layer, and so on are many noticeable MHD applications. Alshomrani and Gul [18] inspected the slight film flow of water-based $\mathrm{Al}_{2} \mathrm{O}_{3}$ and $\mathrm{Cu}$ nanofluid through an extended chamber under the effect of attractive capacity. The characteristics of magneto thermal transport, comprising a time dependent flux of liquid nanofluid thin film flow to a starched surface, were examined by Sandeep and Malvandi [19]. Sandeep [20] examined the characteristics of the hybrid nanolytic flux with various heat and drag forces. Ahmad and Nadeem [21] examined the magnetic effects of hybrid nanofluid with a heat sink/source on micropolitan fluid and achieved numerous findings for hybrid nanofluid and micropolar fluid. Hamrelaine et al. [22] examined the magnetic effect of Jeffery-Hamel flow between nonparallel permeable walls or permeable plates. The attractive impact on the radiative progression of the hybrid nanoliquid thin film with sporadic warmth sink/ source was examined by Anantha Kumar et al. [23]. Zaib et al. [24] got the comparability of various outcomes from magnetite ferroliquid passing on non-Newtonian blood stream with entropy age. Wakif et al. [25] assessed the impact of the magnetic field on progressions of Stokes' second issue with entropy generation. Recently, Kameswaran et al. [26] investigated homogeneous-heterogeneous reactions in nanofluid flow due to a microscopic stretch sheet. They showed that the velocity profiles decrease with an increasing volume of the nanoparticles, while the liquid concentration is reversed by the volume of the nanoparticles for both $\mathrm{Cu}$-water and $\mathrm{Ag}$-water nanofluids.

The cited literature and similar other works show that no study is conducted to examine the combined effects of unstable flow between two squeezing plates in the presence of hybrid nanoparticles. Therefore, using all the studies mentioned above, we analyzed the multifaceted and homogeneous chemical reaction effects on the flow between two compression plates in the presence of hybrid nanoparticles. Navier-Stokes equations, heat transfer, and homogeneous and multifaceted reactions are solved by the HAM and BVP4c. In this work, we analyzed, discussed, and obtained the effects of different parameters on velocity, temperature, concentration, skin friction coefficient, and Nusselt and Sherwood numbers through graphs and tables.

\section{Mathematical Formulation}

Figure 1 shows a laminar, unsteady incompressible, and twodimensional and hybrid nanofluid flowing between horizontally parallel and squeezing plates with homogeneous and heterogeneous reactions. Hybrid nanoparticles comprise $\mathrm{Fe}_{3} \mathrm{O}_{4}$ and $\mathrm{Fe}_{3} \mathrm{O}_{4}+\mathrm{Co}$ in the ethylene glycol with water as base fluid suspension. The plates are separated by a 


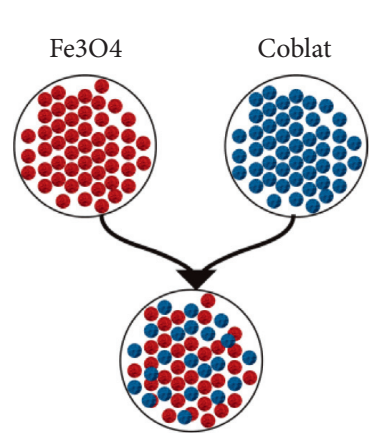

HybirdNanofluids

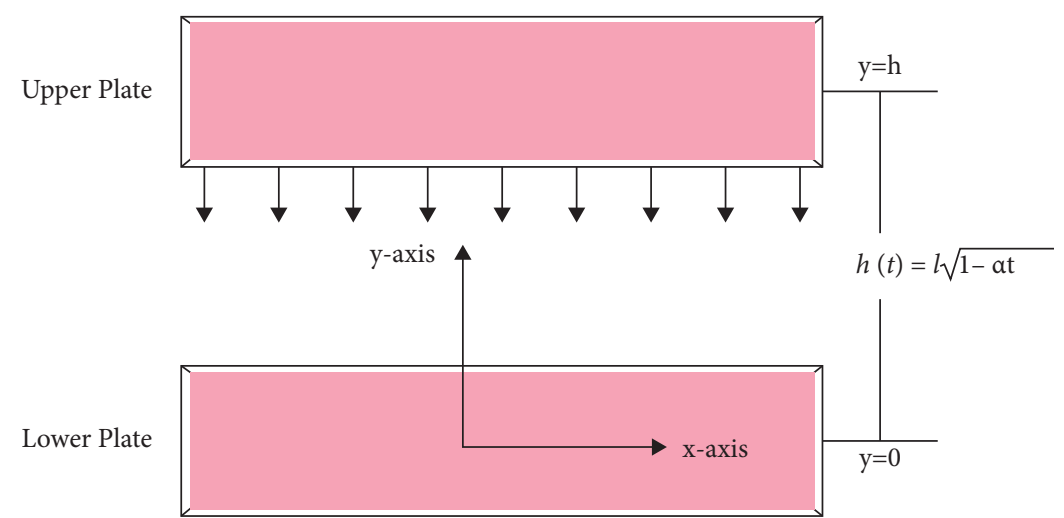

FIgURE 1: Geometrical view of flow problem. gap of $h(t)=l \sqrt{1-\alpha t}$ with $\alpha$ as squeezed parameter. For $\alpha>0$, the plates are squeezing but when $t=(1 / \alpha)$ and $\alpha<0$, the two plates are separated. The velocity field is often impacted by a uniform magnetic field $B(t)=B_{0} / \sqrt{1-\alpha t}$ distributed along the $y$-axis. The upper and lower plates are held at steady temperature $T_{u}$ and $T_{l}$, respectively. The proposed model of Chaudhary and Merkin [27] for homogeneous as well as heterogeneous reaction has been used in this study as described below.

The homogeneous reaction for cubic autocatalyst surface is

$$
N 1+2 N 2 \longrightarrow 3 N 2 \text {. }
$$

The heterogeneous reaction upon catalyst surface is

$$
N 1 \longrightarrow A 2 \text {. }
$$

The quantities of the chemical sorts $N 1$ and $N 2$ are signified by $a$ and $b$, respectively, while the initial conditions are denoted by $k_{c}$. The reaction rate vanishes in exterior flow and beyond the boundary layer bottom, as shown by the equations above. The equations that governed the flow system are presented as [28, 29].

Continuity equation is as follows:

$$
\frac{\partial u}{\partial x}+\frac{\partial v}{\partial y}=0
$$

Navier-Stokes equation is as follows:

$$
\begin{aligned}
& \frac{\partial u}{\partial t}+u \frac{\partial u}{\partial x}+v \frac{\partial u}{\partial y}=-\frac{1}{\rho_{h n f}} \frac{\partial p}{\partial x}+\frac{\mu_{h n f}}{\rho_{h n f}}\left(\frac{\partial^{2} u}{\partial x^{2}}+\frac{\partial^{2} u}{\partial y^{2}}\right)-\sigma_{h n f} B^{2} u \\
& \frac{\partial v}{\partial t}+u \frac{\partial v}{\partial x}+v \frac{\partial v}{\partial y}=-\frac{1}{\rho_{h n f}} \frac{\partial p}{\partial y}+\frac{\mu_{h n f}}{\rho_{h n f}}\left(\frac{\partial^{2} v}{\partial x^{2}}+\frac{\partial^{2} v}{\partial y^{2}}\right) .
\end{aligned}
$$

Energy equation is as follows:

$$
\frac{\partial T}{\partial t}+u \frac{\partial T}{\partial x}+v \frac{\partial T}{\partial y}=\frac{k_{h n f}}{\left(\rho c_{p}\right)_{h n f}}\left(\frac{\partial^{2} T}{\partial x^{2}}+\frac{\partial^{2} T}{\partial y^{2}}\right)+\frac{1}{\left(\rho c_{p}\right)_{h n f}} \frac{16 \sigma^{*} T_{0}^{3}}{3 \kappa^{*}} \frac{\partial^{2} T}{\partial y^{2}}+\frac{Q^{*}}{\left(\rho c_{p}\right)_{h n f}}\left(T-T_{0}\right)
$$

Homogeneous and heterogeneous equations are as follows:

$$
\begin{aligned}
& \frac{\partial a}{\partial t}+u \frac{\partial a}{\partial x}+v \frac{\partial a}{\partial y}=D_{A} \frac{\partial^{2} a}{\partial y^{2}}-K_{c} a b^{2}, \\
& \frac{\partial b}{\partial t}+u \frac{\partial b}{\partial x}+v \frac{\partial b}{\partial y}=D_{B} \frac{\partial^{2} b}{\partial y^{2}}+K_{c} a b^{2} .
\end{aligned}
$$

In the above equations, $T$ represents temperature, $P$ represents pressure, $\rho_{\text {hnf }}$ represents effective density, $\left(\rho c_{p}\right)_{h n f}$ represents effective heat power, and $\sigma_{h n f}$ represents electrical conductivity of nanofluid. The quantities $(u, v)$ denote the fluid's nanofluid velocity component, $T$ is the temperature, and $D_{A}$ and $D_{B}$ are the respective diffusion constants of the chemical sorts $a$ and $b$. The permeability is provided by $K^{*} . k_{h n f}$ nanofluid thermal conductivity, $Q^{*}$ heat generation, $k_{h n f}$ nanofluid thermal conductivity, $k_{h n f}$ nanofluid thermal conductivity, $k_{h n f}$ nanofluid thermal conduct.

2.1. Boundary Conditions. The squeezing flow under consideration has the undermentioned conditions at boundaries:

$$
\begin{aligned}
& u=0, v=0, T=T_{l}, D_{A} \frac{\partial a}{\partial y}=k_{3} a, D_{B} \frac{\partial b}{\partial y}=-k_{3} a \text { at } y=0, \\
& u=0, v=\frac{-\alpha D}{2 \sqrt{1-\alpha t}}, T=T_{u}, a=a_{0}, b=0 \text { at } y=h(t) .
\end{aligned}
$$

Use the transformations [2] 


$$
\begin{aligned}
u & =\frac{\alpha x f^{\prime}(\eta)}{2(1-\alpha t)}, \\
v & =\frac{-\alpha l f(\eta)}{(1-\alpha t)}, \\
T & =\theta(\eta) T_{H}, \\
\eta & =\frac{y}{l \sqrt{1-\alpha t}}, \\
a & =a_{0} H(\eta), \\
b & =a_{0} G(\eta), \\
B(t) & =\frac{B_{0}}{\sqrt{1-a t}}, \\
\theta & =\frac{T-T_{u}}{T_{l}-T_{u}} .
\end{aligned}
$$

Implementing equations (7a) and (7b), we have the following system of equations:

$$
f^{\prime \prime \prime}-S\left(\frac{A_{1}}{A_{4}}\right)\left(\eta f^{\prime \prime \prime}+2 f^{\prime \prime}+f^{\prime \prime} f^{\prime}-f f^{\prime \prime \prime}\right)-\left(\frac{A_{5}}{A_{4}}\right) H a^{2} L f^{\prime \prime}=0,
$$$$
\theta^{\prime \prime}\left(1+\frac{4}{3} R\right)+P_{r} S\left(f \theta^{\prime}-2 \eta \theta+Q \theta\right)=0,
$$$$
H^{\prime \prime}-S_{c} K_{1} H G^{2}-S_{c} S\left(\eta H^{\prime}-f H\right)=0,
$$$$
G^{\prime \prime} \delta-S_{c} K_{1} H G^{2}-S_{c} S\left(\eta G^{\prime}-f G^{\prime}\right)=0,
$$

and the boundary conditions are reduced to

$$
\begin{aligned}
f(0) & =0, f^{\prime}(0)=0, \theta(0)=1, H^{\prime}(0) \\
& =K_{2} H(0), \delta G^{\prime}(0)=-K_{2} H(0), \\
f(1) & =0.5, f^{\prime}(1)=0, \theta(1)=0, H(1)=1, G(1)=0 .
\end{aligned}
$$

Here, $S=\left(\alpha l^{2} / 2 v_{f}\right)$ is the squeezed Reynolds number, $P_{r}=\left(\mu c_{p} / K\right)$ is the Prandtl number, $H a=l B_{0} \sqrt{\sigma_{h n f} / \mu_{f}}$ is the Hartmann number, $R=\left(4 \sigma^{*} T_{0}^{3} / K K^{*}\right)$ is the radiation parameter, $Q=2 Q^{*}(1-\alpha t) / \alpha\left(\rho c_{p}\right)_{h n f}$ is the heat generation parameter, $L=\left(\alpha \sigma_{n f} / x\right)$ is the length parameter, $S_{c}=\left(v_{f} / D_{A}\right)$ is the Schmidt number, $K_{1}=8 k_{c} a_{0}^{2}(1-\alpha t) / \alpha^{3}$ is the homogeneous reaction strength, $K_{2}=\left(k_{s} / D_{A}\right)$ is the heterogeneous reaction strength, and $\delta=\left(D_{A} / D_{B}\right)$ is the ratio of the diffusion coefficients. Here, it is considered that $A$ and $B$ diffusion coefficients of chemical species are of comparable size. The other hypothesis is that $D_{A}$ and $D_{B}$ are equivalent, so $\delta=\left(D_{A} / D_{B}\right)=1$, and $G(\eta)+H(\eta)=1[30]$.

2.2. Coefficients of Interest. The local Nusselt number $(\mathrm{Nu})$, Sherwood number (Sh), and skin friction coefficient (Cf) are some of the coefficients of interest in engineering.

$$
\begin{aligned}
C_{f}^{*} & =\frac{S}{h} C_{f}=f^{\prime \prime}(0), \\
-\theta^{\prime}(0) & =\mathrm{Nu}, \\
-G^{\prime}(0) & =-H^{\prime}(0)=\mathrm{Sh} .
\end{aligned}
$$

By using $A_{i}$ for the dimensionless constant, the result will be as follows:

$$
\begin{aligned}
A_{1}= & \frac{\mu_{n f}}{\mu_{f}}=\frac{1}{(1-\phi)^{2.5}}, \phi=\phi 1+\phi 2, \\
A_{4}= & \frac{\rho_{n f}}{\rho_{f}}=(1-\phi)+\phi 1\left(\frac{\rho_{1}}{\rho_{f}}\right)+\phi 2\left(\frac{\rho_{2}}{\rho_{f}}\right), \\
A_{5}= & \frac{\sigma_{n f}}{\sigma_{f}}=\left(1+3\left(\sigma_{1} \phi_{1}+\sigma_{2} \phi_{2}\right) \sigma_{f}^{-1}-\phi\right) \\
& +\left(\left(\sigma_{1}+\sigma_{2}\right) \sigma_{f}^{-1}+2\right)^{-1}-\left(\left(\sigma_{1} \phi_{1}+\sigma_{2} \phi_{2}\right) \sigma_{f}^{-1}+\phi 1\right)^{-1} .
\end{aligned}
$$

The total volume fraction of nanoparticles is represented by $\phi$. The volume fractions of the discrete nanoparticles are represented by the symbols $\phi 1$ and $\phi 2$; the density of first, second, and base fluids of nanoparticles is $\rho_{1}, \rho_{2}$, and $\rho_{f}$; and the electrical conductivity of 1 st, $2 \mathrm{nd}$, and base fluids of nanoparticles is $\sigma_{1}, \sigma_{2}$ and $\sigma_{f}$.

\section{Approximate Analytical Solution}

To solve system of equations (8)-(11), the analytic method HAM is used. Due to HAM, the functions $f(\varrho), \theta(\varrho), H(\varrho)$, and $G(\varrho)$ can be stated by a set of base functions $\varrho^{c}, c \geq 0$ as

$$
\begin{gathered}
f_{\Psi}(\varrho)=\sum_{\xi=0}^{\infty} a_{\xi} \varrho^{\xi}, \\
\theta_{\Psi}(\varrho)=\sum_{\xi=0}^{\infty} b_{\xi} \varrho^{\xi}, \\
H_{\Psi}(\varrho)=\sum_{\xi=0}^{\infty} c_{\xi} \varrho^{\xi}, \\
G_{\Psi}(\varrho)=\sum_{\xi=0}^{\infty} d_{\xi} \varrho^{\xi},
\end{gathered}
$$

where $a_{\xi}, b_{\xi}, c_{\xi}$, and $d_{\xi}$ are the constant coefficients to be determined. Initial approximations are chosen as follows:

$$
\begin{aligned}
f_{0}(\varrho) & =\frac{3}{2} \varrho^{2}-\varrho^{3}, \\
\theta_{0}(\varrho) & =\left(S_{r}-1\right) \varrho+1, \\
H_{0}(\varrho) & =1-\varrho,
\end{aligned}
$$




$$
G_{0}(\varrho)=1-\varrho .
$$

The auxiliary operators are chosen as

$$
\begin{aligned}
& \ell_{f}=\frac{\partial^{4}}{\partial \varrho^{4}} \\
& \ell_{\theta}=\frac{\partial^{2}}{\partial \varrho^{2}} \\
& \ell_{H}=\frac{\partial^{2}}{\partial \varrho^{2}} \\
& \ell_{G}=\frac{\partial^{2}}{\partial \varrho^{2}}
\end{aligned}
$$

where $\xi_{1}, \xi_{2}, \xi_{3}, \xi_{4}, \xi_{5}, \xi_{6}, \xi_{7}, \xi_{8}, \xi_{9}$, and $\xi_{10}$ are arbitrary constants.

The zeroth order deformation problems can be obtained as

$$
\begin{aligned}
\ell_{f}\left(\xi_{1} \varrho^{3}+\xi_{2} \varrho^{2}+\xi_{3} \varrho+\xi_{4}\right) & =0, \\
\ell_{\theta}\left(\xi_{5} \varrho+\xi_{6}\right) & =0, \\
\ell_{H}\left(\xi_{7} \varrho+\xi_{8}\right) & =0, \\
\ell_{G}\left(\xi_{9} \varrho+\xi_{10}\right) & =0,
\end{aligned}
$$
as

$$
\begin{aligned}
& (1 ; \bowtie) \ell_{f}\left[\bar{f}(\varrho ; \varpi)-f_{0}(\varrho)\right]=q \hbar_{f} N_{f}[\bar{f}(\varrho ; \varpi), \bar{\theta}(\varrho ; \varpi), \bar{m}(\varrho ; \varpi), \bar{n}(\varrho ; \varpi)], \\
& (1 ; @) \ell_{\theta}\left[\bar{\theta}(\varrho ; \varpi)-\theta_{0}(\varrho)\right]=q \hbar_{\theta} N_{\theta}[\bar{f}(\varrho ; @), \bar{\theta}(\varrho ; \varpi), \bar{m}(\varrho ; \varpi), \bar{n}(\varrho ; \varpi)], \\
& (1 ; \varpi) \ell_{H}\left[\bar{H}(\varrho ; \varpi)-H_{0}(\varrho)\right]=q \hbar_{H} N_{H}[\bar{f}(\varrho ; \varpi), \bar{H}(\varrho ; \varpi), \bar{G}(\varrho ; \varpi)], \\
& (1 ; @) \ell_{G}\left[\bar{G}(\varrho ; \emptyset)-G_{0}(\varrho)\right]=q \hbar_{G} N_{G}[\bar{f}(\varrho ; \varpi), \bar{H}(\varrho ; q), \bar{G}(\varrho ; \emptyset)] .
\end{aligned}
$$

with the following properties:

The nonlinear operators of (17)-(20) are defined as

$$
\begin{aligned}
& N_{f}[\bar{f}(\varrho ; \Phi), \bar{\theta}(\varrho ; \varpi)]=\frac{\partial^{4} \bar{f}(\varrho ; \varpi)}{\partial \varrho^{4}}-S\left[\varrho \frac{\partial^{3} \bar{f}(\varrho ; \varpi)}{\partial \varrho^{3}}+2 \frac{\partial^{2} \bar{f}(\varrho ; \varpi)}{\partial \varrho^{2}}+\frac{\partial^{2} \bar{f}(\varrho ; \varpi)}{\partial \varrho^{2}} \frac{\partial \bar{f}(\varrho ; \varpi)}{\partial \varrho}-f \frac{\partial^{3} \bar{f}(\varrho ; \varpi)}{\partial \varrho^{3}}\right] \\
& -H a^{2} L \frac{\partial^{2} \bar{f}(\varrho ; \varpi)}{\partial \varrho^{2}} \\
& N_{\theta}[\bar{f}(\varrho ; \varpi), \bar{\theta}(\varrho ; \varpi)]=\frac{\partial^{2} \bar{\theta}(\varrho ; \varpi)}{\partial \varrho^{2}}\left(1+\frac{4}{3} R\right)+\operatorname{Pr} S\left[f \frac{\partial \bar{\theta}(\varrho ; \varpi)}{\partial \varrho}-2 \varrho \bar{\theta}(\varrho ; \varpi)+Q \bar{\theta}(\varrho ; \varpi)\right] \\
& N_{H}[\bar{f}(\varrho ; \varpi), \bar{H}(\varrho ; \varpi), \bar{G}(\varrho ; \varpi)]=\frac{\partial^{2} \bar{H}(\varrho ; \varpi)}{\partial \varrho^{2}}-S_{c} K_{1} \bar{H}(\varrho ; \varpi) \frac{\partial^{2} \bar{G}(\varrho ; \varpi)}{\partial \varrho^{2}}-S_{c} S\left[\varrho \frac{\partial \bar{H}(\varrho ; \varpi)}{\partial \varrho}-f \bar{H}(\varrho ; \varpi)\right],
\end{aligned}
$$

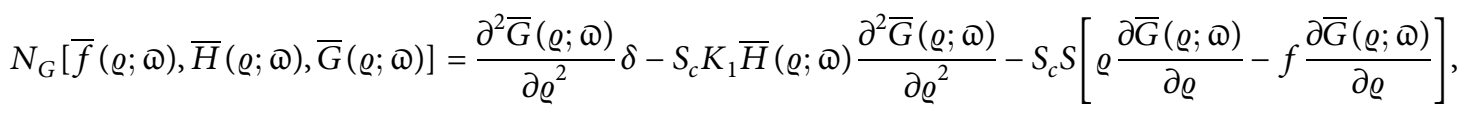

where $₫$ is an embedding parameter; $\hbar_{f}, \hbar_{\theta}, \hbar_{H}$, and $\hbar_{G}$ are the nonzero auxiliary parameter; and $N_{f}, N_{\theta}, N_{H}$, and $N_{G}$ are the nonlinear parameters. 
For $\omega=0$ and 1 , we have

$$
\begin{aligned}
& \bar{f}(\varrho, 0)=f_{o}(\varrho), \bar{f}(\varrho, 1)=f(\varrho), \\
& \bar{\theta}(\varrho, 0)=\theta_{o}(\varrho), \bar{\theta}(\varrho, 1)=\theta(\varrho), \\
& \bar{H}(\varrho, 0)=H_{o}(\varrho), \bar{H}(\varrho, 1)=H(\varrho), \\
& \bar{G}(\varrho, 0)=G_{o}(\varrho), \bar{G}(\varrho, 1)=G(\varrho),
\end{aligned}
$$

so we can say that as $\varrho$ varies from 0 to $1, \bar{f}(\varrho, 0), \bar{\theta}(\varrho, 0)$, $\bar{H}(\varrho, 0)$, and $\bar{G}(\varrho, 0)$ vary from initial guesses $f_{0}(\varrho), \theta_{0}(\varrho)$, $H_{0}(\varrho)$, and $G_{0}(\varrho)$ to exact solutions $f(\varrho), \theta(\varrho), H(\varrho)$, and $G(\varrho)$, respectively.

Taylor's series expansion of these functions yields

$$
\begin{aligned}
& f(\varrho ; \varpi)=f_{0}(\varrho)+\sum_{\Psi=1}^{\infty} \varrho^{\Psi} f_{\Psi}(\varrho), \\
& \theta(\varrho ; \Phi)=\theta_{0}(\varrho)+\sum_{\Psi=1}^{\infty} \Phi^{\Psi} \theta_{\Psi}(\varrho), \\
& H(\varrho ; \varpi)=H_{0}(\varrho)+\sum_{\Psi=1}^{\infty}{\Phi^{\Psi}}^{\Psi}(\varrho) \text {, }
\end{aligned}
$$

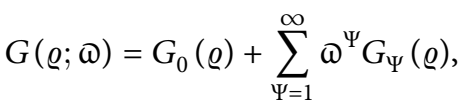

$$
\begin{aligned}
& f_{\Psi}(\varrho)=\left.\frac{1}{\Psi !} \frac{\partial^{\Psi} f(\varrho ; \Phi)}{\partial \varrho^{\Psi}}\right|_{\varpi=0}, \\
& \theta_{\Psi}(\varrho)=\left.\frac{1}{\Psi !} \frac{\partial^{\Psi} \theta(\varrho ; \Phi)}{\partial \varrho^{\Psi}}\right|_{\varpi=0}, \\
& H_{\Psi}(\varrho)=\left.\frac{1}{\Psi !} \frac{\partial^{\Psi} H(\varrho ; \Phi)}{\partial \varrho^{\Psi}}\right|_{\varpi=0}, \\
& G_{\Psi}(\varrho)=\left.\frac{1}{\Psi !} \frac{\partial^{\Psi} G(\varrho ; \Phi)}{\partial \varrho^{\Psi}}\right|_{\varpi=0} .
\end{aligned}
$$

$$
\begin{aligned}
& R_{f, \Psi}(\varrho)=f_{\Psi-1}^{\prime \prime}(\varrho)-S\left(\frac{A_{1}}{A_{4}}\right)\left[\varrho f_{\Psi-1}^{\prime \prime}(\varrho)+2 f_{\Psi-1}^{\prime \prime}(\varrho)+2 \sum_{j=0}^{\Psi-1} f_{j}(\varrho) f_{\Psi-j-1}^{\prime \prime \prime}(\varrho)-\sum_{j=0}^{\Psi-1} f_{j}(\varrho) f_{\Psi-j-1}^{\prime \prime}(\varrho)\right]-\left(\frac{A_{5}}{A_{4}}\right) H a^{2} L f_{\Psi-1}^{\prime \prime}(\varrho), \\
& R_{\theta, \Psi}(\varrho)=\theta_{\Psi-1}^{\prime \prime}(\varrho)\left(1+\frac{4}{3} R\right)+\operatorname{Pr} S\left[\sum_{j=0}^{\Psi-1} f_{j}(\varrho) \theta_{\Psi-j-1}^{\prime}(\varrho)-2 \varrho \theta_{\Psi-1}(\varrho)+Q \theta_{\Psi-1}(\varrho)\right] \\
& R_{H, \Psi}(\varrho)=H_{\Psi-1}^{\prime \prime}(\varrho)+S_{c} K_{1} \sum_{j=0}^{\Psi-1} H_{j}(\varrho) G_{\Psi-j-1}^{\prime \prime}(\varrho)-S_{c} S\left[\varrho H_{\Psi-1}^{\prime}(\varrho)-\sum_{j=0}^{\Psi-1} f_{j}(\varrho) H_{\Psi-j-1}(\varrho)\right] \\
& R_{G, \Psi}(\varrho)=G_{\Psi-1}^{\prime \prime}(\varrho) \delta-S_{c} K_{1} \sum_{j=0}^{\Psi-1} H_{j}(\varrho) G_{\Psi-j-1}^{2}(\varrho)-S_{c} S\left[\varrho G_{\Psi-1}^{\prime}(\varrho)-\sum_{j=0}^{\Psi-1} f_{j}(\varrho) G_{\Psi-j-1}^{\prime}(\varrho)\right]
\end{aligned}
$$

and $\chi_{\Psi}=\{1$, if $\Psi>1$, and 0 , if $\Psi=1\}$. .

For $\omega=1$, we have

$$
\begin{aligned}
& f(\varrho)=f_{0}(\varrho)+\sum_{\Psi=1}^{\infty} f_{\Psi}(\varrho), \\
& \theta(\varrho)=\theta_{0}(\varrho)+\sum_{\Psi=1}^{\infty} \theta_{\Psi}(\varrho), \\
& H(\varrho)=H_{0}(\varrho)+\sum_{\Psi=1}^{\infty} H_{\Psi}(\varrho), \\
& G(\varrho)=G_{0}(\varrho)+\sum_{\Psi=1}^{\infty} G_{\Psi}(\varrho) .
\end{aligned}
$$

subject to the boundary conditions

$$
\begin{aligned}
f_{\Psi}(0) & =0, f_{\Psi}^{\prime}(0)=0, \theta_{\Psi}(0)=1, \\
H_{\Psi}^{\prime}(0) & =k_{2} H(0), \delta G_{\Psi}^{\prime}(0)=-k_{2} H(0), \\
f_{\Psi}(1) & =0.5, f_{\Psi}^{\prime}(1)=0, \theta_{\Psi}(1)=0, \\
H_{\Psi}(1) & =1, G f_{\Psi}^{\prime}(1)=0,
\end{aligned}
$$

where

$$
\begin{gathered}
\ell_{f}\left[f_{\Psi}(\varrho)-\chi_{\Psi} f_{\Psi-1}(\varrho)\right]=\hbar_{f} R_{f, \Psi}(\varrho), \\
\ell_{\theta}\left[\theta_{\Psi}(\varrho)-\chi_{\Psi} \theta_{\Psi-1}(\varrho)\right]=\hbar_{\theta} R_{g, \Psi}(\varrho), \\
\ell_{H}\left[H_{\Psi}(\varrho)-\chi_{\Psi} H_{\Psi-1}(\varrho)\right]=\hbar_{H} R_{H, \Psi}(\varrho), \\
\ell_{G}\left[G_{\Psi}(\varrho)-\chi_{\Psi} G_{\Psi-1}(\varrho)\right]=\hbar_{\phi} R_{G, \Psi}(\varrho),
\end{gathered}
$$


Finally, the general solution of (36)-(39) can be written as

$$
\begin{aligned}
& f_{\Psi}(\varrho)=\int_{0}^{\varrho} \int_{0}^{\varrho} \int_{0}^{\varrho} \int_{0}^{\varrho} \hbar_{f} R_{f, \Psi}(z) \mathrm{d} z \mathrm{~d} z \mathrm{~d} z \mathrm{~d} z+\chi_{\Psi} f_{\Psi-1}+\xi_{1} \varrho^{3}+\xi_{2} \varrho^{2}+\xi_{3} \varrho+\xi_{4}, \\
& \theta_{\Psi}(\varrho)=\int_{0}^{\varrho} \int_{0}^{\varrho} \hbar_{\theta} R_{\theta, \Psi}(z) \mathrm{d} z \mathrm{~d} z+\chi_{\Psi} \theta_{\Psi-1}+\xi_{5} \varrho+\xi_{6}, \\
& H_{\Psi}(\varrho)=\int_{0}^{\varrho} \int_{0}^{\varrho} \hbar_{H} R_{H, \Psi}(z) \mathrm{d} z \mathrm{~d} z+\chi_{\Psi} H_{\Psi-1}+\xi_{11} \varrho+\xi_{12}, \\
& G_{\Psi}(\varrho)=\int_{0}^{\varrho} \int_{0}^{\varrho} \hbar_{G} R_{G, \Psi}(z) \mathrm{d} z \mathrm{~d} z+\chi_{\Psi} G_{\Psi-1}+\xi_{13} \varrho+\xi_{14},
\end{aligned}
$$

and so the exact solutions $f(\varrho), \theta(\varrho), H(\varrho)$, and $H(\varrho)$ become

$$
\begin{gathered}
f(\varrho) \approx \sum_{n=0}^{\Psi} f_{n}(\varrho), \\
\theta(\varrho) \approx \sum_{n=0}^{\Psi} \theta_{n}(\varrho), \\
H(\varrho) \approx \sum_{n=0}^{\Psi} H_{n}(\varrho), \\
G(\varrho) \approx \sum_{n=0}^{\Psi} G_{n}(\varrho) .
\end{gathered}
$$

\section{Optimizing the Convergence of Control Parameter}

It is important to note that the series solutions (46)-(49) include $\hbar_{f}, \hbar_{\theta}, \hbar_{H}$, and $\hbar_{G}$ nonzero auxiliary parameters that define the convergence area as well as rate of the homotopy series solutions. The residual error to obtain the maximum values of $\hbar_{f}, \hbar_{\theta}, \hbar_{H}$, and $\hbar_{G}$ was used as

$$
\begin{aligned}
& \epsilon_{\Psi}^{f}=\frac{1}{\xi+1} \sum_{j=0}^{\xi}\left[N_{f} \sum_{i=0}^{\Psi} \bar{f}(\varrho), \sum_{i=0}^{\Psi} \bar{\theta}(\varrho)\right]^{2} \mathrm{~d} \varrho \\
& \epsilon_{\Psi}^{\theta}=\frac{1}{\xi+1} \sum_{j=0}^{\xi}\left[N_{\theta}\left(\sum_{i=0}^{\Psi} \bar{f}(\varrho), \sum_{i=0}^{\Psi} \bar{\theta}(\varrho)\right)_{n=j D_{u} n}\right]^{2} \mathrm{~d} \varrho, \\
& \epsilon_{\Psi}^{H}=\frac{1}{\xi+1} \sum_{j=0}^{\xi}\left[N_{H}\left(\sum_{i=0}^{\Psi} \bar{f}(\varrho), \sum_{i=0}^{\Psi} \bar{H}(\varrho), \sum_{i=0}^{\Psi} \bar{G}(\varrho)\right)_{n=j D_{u} n}\right]^{2} \mathrm{~d} \varrho, \\
& \epsilon_{\Psi}^{G}=\frac{1}{\xi+1} \sum_{j=0}^{\xi}\left[N_{G}\left(\sum_{i=0}^{\Psi} \bar{f}(\varrho), \varrho \sum_{i=0}^{\Psi} \bar{H}(\varrho), \sum_{i=0}^{\Psi} \bar{G}(\varrho)\right)_{n=j D_{u} n}\right]^{2} \mathrm{~d} \varrho .
\end{aligned}
$$

Due to Liao

$$
\epsilon_{\Psi}^{t}=\epsilon_{\Psi}^{f}+\epsilon_{\Psi}^{\theta}+\epsilon_{\Psi}^{H}+\epsilon_{\Psi}^{G},
$$

where $\epsilon_{\Psi}^{t}$ is the total squared residual error.

\section{Analysis of Error}

An error analysis is conducted to ensure a minimum residual error for the efficiency of the analysis. HAM and BVP4c solve the problem analytically and numerically. The analysis is conducted using an approximation order of the 40th order. For this analysis, the validity of HAM techniques is also evaluated using the Mathematica software BVPh 2.0 for maximum residual error $10^{-40}$. The results are compared with the numerical solution of BVP4c using Matlab for the authentication and consistency of the HAM solution. The reliability of the two methods for various concerning physical parameters is investigated by error analysis in Figure 2 and Tables $1-12$. Figure 2 shows that up to the 16 th transition series, the maximum average residual errors of $f^{\prime \prime}(\eta),-\theta^{\prime}(\eta),-H^{\prime}(\eta)$, and $-G^{\prime}(\eta)$ are almost gradually decreased. The cumulative residual error for the various approximation orders of fixed $P_{r}=-0.5, S=-0.5, L=0.01$, $Q=5, R=0.7, \delta=1, S c=2.5, K 1=0.1, K 2=1$, and $H a=$ 0.1 values is shown in Table 1 . Table 2 shows various orders of approximation with distinct average squared residual error for $f^{\prime \prime}(\eta),-\theta^{\prime}(\eta),-H^{\prime}(\eta)$, and $-G^{\prime}(\eta)$ with fixed values as $P_{r}=0.5, S=-0.5, L=0.01, \delta=1, Q=0.01$, $R=0.1, S c=2.5, K 1=0.3, K 2=0.1, H a=0.2 \phi=0.02$, $\phi 1=\phi 2=0.01, \quad \rho 1=5180, \quad \rho 2=8900, \quad \rho 3=1056$, $\sigma 1=0.74 \times 10^{6}, \sigma 2=1.602 \times 10^{7}, \sigma 3=0.00509$. The comparison of the analytical and numerical values by HAM and BVP4c is shown in Table 3 for various values of $\eta$ and fixed values of other parameters, $P_{r}=0.5, S=-0.5, L=0.01$, $\delta=1, \quad Q=0.01, \quad R=0.1, \quad S c=2.5, \quad K 1=0.3, \quad K 2=0.1$, $H a=0.2, \phi=0.02, \phi 1=\phi 2=0.01, \rho 1=5180, \rho 2=8900$, $\rho 3=1056, \quad \sigma 1=0.74 \times 10^{6}, \quad \sigma 2=1.602 \times 10^{7}, \quad$ and $\sigma 3=0.00509$.

\section{Results and Discussion}

For various values of parameters $S, H a, P_{r}, Q, R, S c, K 1, K 2$, and $\phi$, the system of nonlinear equations (8)-(11), resulting in the boundary conditions equation (12), is numerically 

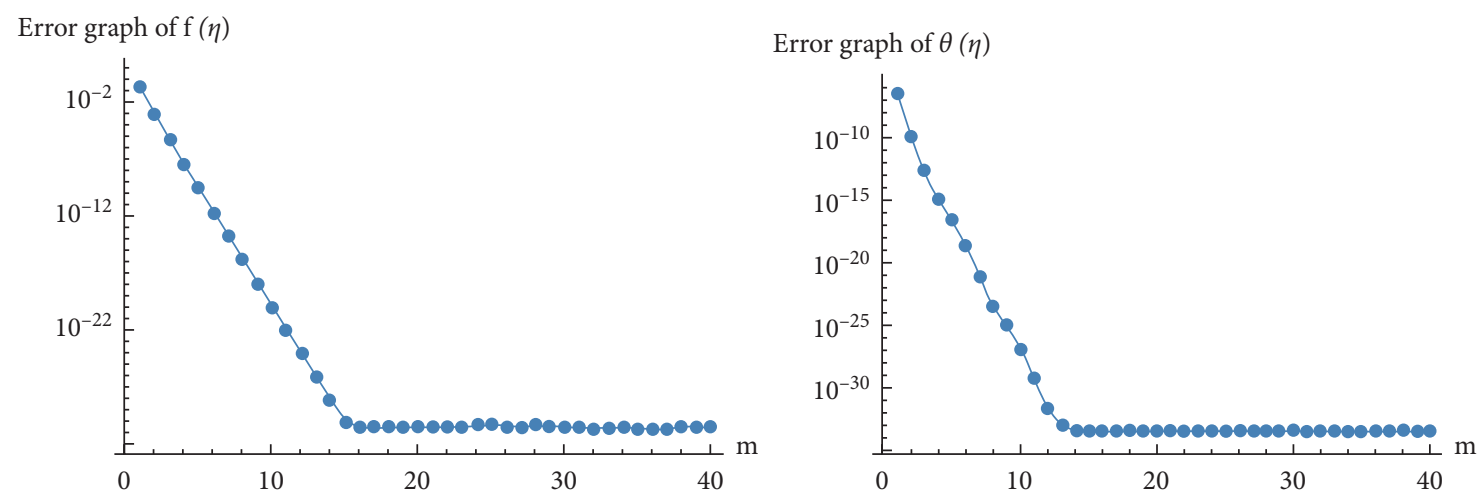

Error graph of $\mathrm{H}(\eta)$

Error graph of $\mathrm{G}(\eta)$
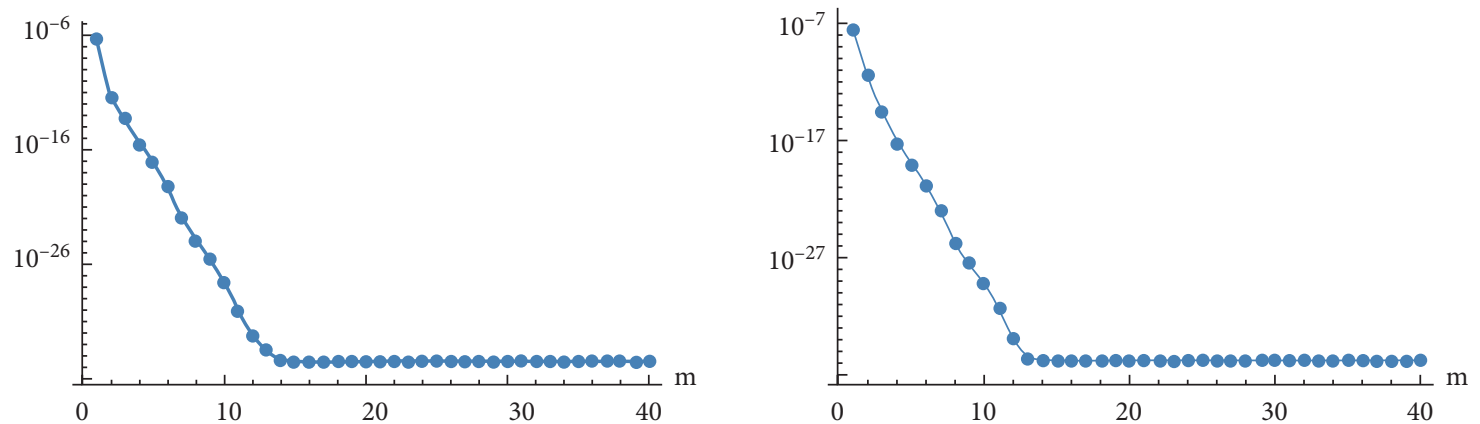

Figure 2: Error analysis on $f(\eta), \theta(\eta), G(\eta)$, and $H(\eta)$ with $P_{r}=-0.5, S=-0.5, L=0.01, Q=5, R=0.7, \delta=1, S c=2.5, K 1=0.1, K 2=1$, and $H a=0.1$.

TABLE 1: Thermophysical properties of iron oxide, cobalt, and ethylene glycol with water at $20^{\circ} \mathrm{C}$ [7].

\begin{tabular}{|c|c|c|c|c|c|}
\hline Physical properties & $\rho\left(\mathrm{kg} / \mathrm{m}^{3}\right)$ & $c_{p}(\mathrm{~J} / \mathrm{kgK})$ & $k(\mathrm{~W} / \mathrm{mK})$ & $\beta \times 10^{-5}\left(K^{-1}\right)$ & $\sigma\left(\mathrm{sm}^{-1}\right)$ \\
\hline $\mathrm{Fe}_{3} \mathrm{O}_{4}$ & 5180 & 670 & 9.7 & 1.3 & $0.74 \times 10^{6}$ \\
\hline Co (cobalt) & 8900 & 420 & 100 & $1.3 \times 10^{-5}$ & $1.602 \times 10^{7}$ \\
\hline $\mathrm{C}_{2} \mathrm{H}_{6} \mathrm{O}_{2}-\mathrm{H}_{2} \mathrm{O}$ & 1056 & 3630 & 0.387 & 58 & 0.00509 \\
\hline
\end{tabular}

TABLE 2: Total residual error of $f(\eta), \theta(\eta), G(\eta)$, and $H(\eta)$ with $P_{r}=-0.5, S=-0.5, L=0.01, Q=5, R=0.7, \delta=1, S c=2.5, K 1=0.1$, $K 2=1$, and $H a=0.1$

\begin{tabular}{lcccc}
\hline$n$ & $\epsilon^{f} n$ & $\epsilon^{\theta} n$ & $\epsilon^{H} n$ & $\epsilon^{G} n$ \\
\hline 1 & 0.186129 & $2.4373 \times 10^{-7}$ & $3.17987 \times 10^{-7}$ & $2.30094 \times 10^{-9}$ \\
5 & $2.936895 \times 10^{-10}$ & $2.30705 \times 10^{-17}$ & $7.84948 \times 10^{-18}$ & $8.67775 \times 10^{-20}$ \\
10 & $1.34057 \times 10^{-20}$ & $1.16169 \times 10^{-27}$ & $3.02765 \times 10^{-28}$ & $6.44809 \times 10^{-30}$ \\
15 & $1.3174 \times 10^{-30}$ & $3.18812 \times 10^{-34}$ & $3.27408 \times 10^{-35}$ & $1.86574 \times 10^{-36}$ \\
16 & $3.64848 \times 10^{-31}$ & $3.20099 \times 10^{-34}$ & $3.17778 \times 10^{-35}$ & $1.86574 \times 10^{-36}$ \\
20 & $3.64848 \times 10^{-31}$ & $3.20099 \times 10^{-34}$ & $3.17778 \times 10^{-35}$ & $1.86574 \times 10^{-36}$ \\
25 & $3.64848 \times 10^{-31}$ & $3.20099 \times 10^{-34}$ & $3.17778 \times 10^{-35}$ & $1.86574 \times 10^{-36}$ \\
30 & $3.64848 \times 10^{-31}$ & $3.20099 \times 10^{-34}$ & $3.17778 \times 10^{-35}$ & $1.86574 \times 10^{-36}$ \\
35 & $3.64848 \times 10^{-31}$ & $3.20099 \times 10^{-34}$ & $1.86574 \times 10^{-36}$ \\
40 & $3.64848 \times 10^{-31}$ & $3.20099 \times 10^{-34}$ & $3.17778 \times 10^{-35}$ & $1.86574 \times 10^{-36}$ \\
\hline
\end{tabular}

resolved using HAM and BVP4c numerical routines. The local skin friction coefficient as well as the local rates of heat and mass transfer at the surface of the squeezing plates, both of which are extremely important in terms of physical properties, is also computed. Because there are so many physical parameters in the current problem, a wide range of results can be obtained. The distributions of temperature, velocity, pressure, and mass transfer are obtained by solving
(8)-(11) and are shown in Figures 1-10 for various physical parameters. The impact of the squeeze number $S$ on the velocity and temperature distribution for both heating and cooling surfaces is plotted on $f(\eta), f^{\prime}(\eta), \theta(\eta), H(\eta)$, and $G(\eta)$ in Figure 5 in the case of both $\mathrm{Fe}_{3} \mathrm{O}_{4}-\mathrm{C}_{2} \mathrm{H}_{6} \mathrm{O}_{2}-\mathrm{H}_{2} \mathrm{O}$ single nanofluids (SNF) and $\mathrm{Fe}_{3} \mathrm{O}_{4}-\mathrm{Co} / \mathrm{C}_{2} \mathrm{H}_{6} \mathrm{O}_{2}-\mathrm{H}_{2} \mathrm{O}$ hybrid nanofluids (HNF). The values of the squeeze number have been set to be higher than zero in this case, indicating 
TABLE 3: Computation of nanoparticles (iron oxide, cobalt) and ethylene glycol for $f(\eta), \theta(\eta), H(\eta)$, and $G(\eta)$ with $P_{r}=0.5, S=-0.5$, $L=0.01, \delta=1, Q=0.01, R=0.1, S c=2.5, K 1=0.3, K 2=0.1, H a=0.2, \phi=0.02, \phi 1=\phi 2=0.01, \rho 1=5180, \rho 2=8900, \rho 3=1056$, $\sigma 1=0.74 \times 10^{6}, \sigma 2=1.602 \times 10^{7}$, and $\sigma 3=0.00509$.

\begin{tabular}{lccccccc}
\hline & \multicolumn{3}{c}{ HAM results } & \multicolumn{3}{c}{ Numerical results } \\
$\eta$ & $f(\eta)$ & $\theta(\eta)$ & $H(\eta)$ & $G(\eta)$ & $f(\eta)$ & $\theta(\eta)$ & $H(\eta)$ \\
\hline 0.0000 & 0.0000 & 1.0000 & 1.0193 & 0.1242 & 0.0000 & 1.0000 & 1.0193 \\
0.1001 & 0.0146 & 0.9229 & 1.0308 & 0.1106 & 0.0146 & 0.9229 & 1.0308 \\
0.2002 & 0.0542 & 0.8441 & 1.0447 & 0.0966 & 0.0542 & 0.8441 & 1.0447 \\
0.3003 & 0.1122 & 0.7621 & 1.0602 & 0.0825 & 0.1122 & 0.7621 & 1.0602 \\
0.4004 & 0.1820 & 0.6752 & 1.0757 & 0.0685 & 0.1820 & 0.6752 & 1.0757 \\
0.5005 & 0.2570 & 0.5822 & 1.0892 & 0.0550 & 0.2570 & 0.5822 & 1.0892 \\
0.6006 & 0.3310 & 0.4820 & 1.0980 & 0.0422 & 0.3310 & 0.4820 & 0.0966 \\
0.7007 & 0.3978 & 0.3736 & 1.0986 & 0.0301 & 0.3978 & 0.3736 & 0.0685 \\
0.8008 & 0.4517 & 0.2567 & 1.0865 & 0.0189 & 0.4517 & 0.2567 & 1.0986 \\
0.9009 & 0.4873 & 0.1315 & 1.0560 & 0.0088 & 0.4873 & 0.1315 & 0.0422 \\
0.0000 & 0.5000 & 0.0000 & 1.0000 & 0.0000 & 0.5000 & 0.0000 & 0.0301 \\
\hline
\end{tabular}

TABLE 4: Convergence of homotopy solution for different orders of calculation of nanoparticles (iron oxide, cobalt) and ethylene glycol for $f^{\prime \prime}(\eta),-\theta^{\prime}(\eta),-H^{\prime}(\eta)$, and $-G^{\prime}(\eta)$ with $P_{r}=0.5, S=-0.5, L=0.01, \delta=1, Q=0.01, R=0.1, S c=2.5, K 1=0.3, K 2=0.1, H a=0.2$, $\phi=0.02, \phi 1=\phi 2=0.01, \rho 1=5180, \rho 2=8900, \rho 3=1056, \sigma 1=0.74 \times 10^{6}, \sigma 2=1.602 \times 10^{7}$, and $\sigma 3=0.00509$.

\begin{tabular}{lcccc}
\hline$\eta$ & $f^{\prime \prime}(0)$ & $-\theta^{\prime}(0)$ & $-H^{\prime}(0)$ & $-G^{\prime}(0)$ \\
\hline 0.0000 & 3.1331 & 0.7679 & -0.1019 & 0.1019 \\
0.1001 & 2.4908 & 0.7758 & -0.1279 & 0.0911 \\
0.2002 & 1.8362 & 0.8006 & -0.1486 & 0.0800 \\
0.3003 & 1.1779 & 0.8413 & -0.1576 & 0.0689 \\
0.4004 & 0.5255 & 0.8963 & -0.1490 & 0.0579 \\
0.5005 & -0.1106 & 0.9637 & 0.1166 & 0.0472 \\
0.6066 & -0.7555 & 1.0459 & 0.0484 & 0.0362 \\
0.7007 & -1.2931 & 1.1247 & 0.0492 & 0.0268 \\
0.8008 & -1.8216 & 1.2101 & 0.2020 & 0.0173 \\
0.9009 & -2.2987 & 1.2912 & 0.4208 & 0.0082 \\
1.0000 & -2.7161 & 1.3595 & 0.7259 & 0.0000 \\
\hline
\end{tabular}

TABLE 5: Computation of nanoparticles (iron oxide and ethylene glycol) and hybrid nanoparticles (iron oxide, cobalt, and ethylene glycol with water) for skin friction $f^{\prime \prime}(0)$.

\begin{tabular}{lcccc}
\hline Different fluid model parameters & & $\begin{array}{c}\text { Nanoparticles } \\
f^{\prime \prime}(0)\end{array}$ & $\begin{array}{c}\text { Hybrid nanoparticles } \\
f^{\prime \prime}(0)\end{array}$ \\
\hline-0.5 & $H a$ & $\phi$ & 0.057180 & 3.083700 \\
-1 & 1 & 0.1 & 0.053850 & 3.077570 \\
-1.5 & & & 0.050680 & 3.072110 \\
-2 & 1 & & 3.057180 & 3.028170 \\
& 3 & & 3.057180 & 3.028170 \\
& 5 & & 3.057180 & 3.028170 \\
& 7 & 0.1 & 3.019960 & 3.029040 \\
& & 0.2 & 3.019620 & 3.028170 \\
\hline
\end{tabular}

that the upper plate is moving away from the lower stationary plate and the opposite inequality of the squeeze number indicating that the lower plate is moving away from the upper plate. Effect of $S$ on $f(\eta)$ and $f^{\prime}(\eta)$ is depicted in Figure 3 in the case of both SNF and HNF. In reality, for larger $S$, the upper plate slides downward, which puts more stress on nanoparticles, and consequently velocity components $f(\eta), f^{\prime}(\eta)$ are amplified. Effect of $S$ on $\theta(\eta)$ is demonstrated in Figure 3. For bigger $S$, the top plate shift downwards, and interatomic collision nanoparticles increase; hence, the temperature increases. The influence on concentration profiles $H(\eta)$ and $G(\eta)$ of the parameter $S$ can be observed in Figure 3 in both SNF and HNF. The concentration profile $H(\eta)$ decreases and $G(\eta)$ increases. It has been discovered that as $S$ increases, the homogeneous chemical reaction increases, resulting in a decrease in viscosity. However, the $H(\eta)$ indicates the reverse of the $G(\eta)$ above can seen in Figure 3. The impact of Hartmann number $H a$ show in Figure 3 on $f(\eta)$ against the similarity variable $\eta$ for the phenomenon of both SNF and HNF are decreases. When the HNF flow is applied, the fluid velocity is reduced by moving down the horizontal axis and the SNF flow 
TABLE 6: Computation of nanoparticles (iron oxide and ethylene glycol) and hybrid nanoparticles (iron oxide, cobalt) and ethylene glycol with water for Nusselt number $-\theta^{\prime}(0)$.

\begin{tabular}{|c|c|c|c|c|c|c|}
\hline \multirow[b]{2}{*}{$S$} & \multicolumn{4}{|c|}{ Different fluid model parameters } & \multirow{2}{*}{$\begin{array}{l}\text { Nanoparticles } \\
-\theta^{\prime}(0)\end{array}$} & \multirow{2}{*}{$\begin{array}{l}\text { Hybrid nanoparticles } \\
-\theta^{\prime}(0)\end{array}$} \\
\hline & $P_{r}$ & Q & $R$ & $\phi$ & & \\
\hline-0.5 & 1 & 1 & 1 & 0.1 & 1.101390 & 1.101380 \\
\hline-1 & & & & & 1.201800 & 1.201760 \\
\hline-1.5 & & & & & 1.301230 & 1.301160 \\
\hline \multirow[t]{12}{*}{-2} & 1 & & & & 1.201810 & 1.201790 \\
\hline & 2 & & & & 1.399750 & 1.399710 \\
\hline & 3 & & & & 1.593820 & 1.593760 \\
\hline & 4 & 1 & & & 1.008540 & 1.008530 \\
\hline & & 2 & & & 1.039600 & 1.039590 \\
\hline & & 3 & & & 1.070550 & 1.070540 \\
\hline & & 4 & 1 & & 1.094100 & 1.094100 \\
\hline & & & 2 & & 1.079550 & 1.079550 \\
\hline & & & 3 & & 1.064990 & 1.064990 \\
\hline & & & 4 & 0.1 & 1.054580 & 1.054580 \\
\hline & & & & 0.2 & 1.054580 & 1.054580 \\
\hline & & & & 0.3 & 1.054580 & 1.054580 \\
\hline
\end{tabular}

TABLE 7: Computation of nanoparticles (iron oxide and ethylene glycol) and hybrid nanoparticles (iron oxide, cobalt) and ethylene glycol with water for Sherwood numbers (homogeneous-heterogeneous) $H^{\prime \prime}(0)$ and $G^{\prime \prime}(0)$.

\begin{tabular}{|c|c|c|c|c|c|c|c|c|}
\hline \multicolumn{5}{|c|}{ Variation in parameters } & \multicolumn{2}{|c|}{ Nanoparticles } & \multicolumn{2}{|c|}{ Hybrid nanoparticles } \\
\hline$S$ & Sc & $k 1$ & $k 2$ & $\phi$ & $-H^{\prime}(0)$ & $-G^{\prime}(0)$ & $-H^{\prime}(0)$ & $-G^{\prime}(0)$ \\
\hline-0.5 & 1 & 0.1 & 1 & 0.1 & -0.333233 & 0.167006 & -0.333230 & 0.167016 \\
\hline-1 & & & & & -0.332977 & 0.167346 & -0.332968 & 0.167332 \\
\hline 0.5 & & & & & -0.332720 & 0.167686 & -0.332700 & 0.167680 \\
\hline-2 & 1 & & & & -0.333233 & 0.167006 & -0.333230 & 0.167005 \\
\hline & 2 & & & & -0.333135 & 0.167346 & -0.333129 & 0.167345 \\
\hline & 3 & & & & -0.333038 & 0.167688 & -0.333029 & 0.167686 \\
\hline & 4 & 0.1 & & & -0.333283 & 0.166396 & -0.333286 & 0.166396 \\
\hline & & 0.2 & & & -0.332976 & 0.166531 & -0.332979 & 0.166532 \\
\hline & & 0.3 & & & -0.332669 & 0.166531 & -0.332672 & 0.166667 \\
\hline & & 0.4 & 1 & & -0.499141 & 0.249089 & -0.499141 & 0.249089 \\
\hline & & & 6 & & -0.852392 & 0.430977 & -0.852392 & 0.430977 \\
\hline & & & 11 & & -0.911060 & 0.460473 & -0.911060 & 0.460473 \\
\hline & & & 4 & 0.1 & -0.333387 & 0.166454 & -0.333387 & 0.166454 \\
\hline & & & & 0.2 & -0.333387 & 0.166454 & -0.333387 & 0.166454 \\
\hline & & & & 0.3 & -0.333387 & 0.166454 & -0.333387 & 0.166454 \\
\hline
\end{tabular}

TABLE 8: Computation for $f^{\prime \prime}(0),-\theta^{\prime}(0),-H^{\prime}(0)$, and $-G^{\prime}(0)$ with $P_{r}=0.5, S=-0.5, L=0.01, \delta=1, Q=0.01, R=0.1, S c=2.5, K 1=0.3$, $K 2=0.1, H a=0.2, \phi=0.02, \phi 1=\phi 2=0.01, \rho 1=5180, \rho 2=8900, \rho 3=1056, \sigma 1=0.74 \times 10^{6}, \sigma 2=1.602 \times 10^{7}$, and $\sigma 3=0.00509$ and varied values of $S$.

\begin{tabular}{llllllll}
\hline & \multicolumn{3}{c}{ HAM results } & \multicolumn{3}{c}{ Numerical results } \\
$S$ & $f^{\prime \prime}(0)$ & $-\theta^{\prime}(0)$ & $-H^{\prime}(0)$ & $-G^{\prime}(0)$ & $f^{\prime \prime}(0)$ & $-\theta^{\prime}(0)$ & $-H^{\prime}(0)$ \\
\hline-1.1 & 3.1283 & 0.8762 & -0.1209 & 0.1209 & 3.1283 & 0.8762 & -0.1209 \\
-1.2 & 3.1357 & 0.8641 & -0.1324 & 0.1324 & 3.1357 & 0.8641 & -0.1324 \\
-1.3 & 3.1443 & 0.8519 & -0.1411 & 0.1411 & 3.1443 & 0.8519 & -0.1411 \\
-1.4 & 3.1540 & 0.8395 & -0.1515 & 0.1515 & 3.1540 & 0.8395 & -0.1515 \\
\hline
\end{tabular}

TABle 9: Computation for $f^{\prime \prime}(0),-\theta^{\prime}(0),-H^{\prime}(0)$, and $-G^{\prime}(0)$ with $P_{r}=0.5, S=-0.5, L=0.01, \delta=1, Q=0.01, R=0.1, S c=2.5, K 1=0.3$, $K 2=0.1, H a=0.2, \phi=0.02, \phi 1=\phi 2=0.01, \rho 1=5180, \rho 2=8900, \rho 3=1056, \sigma 1=0.74 \times 10^{6}, \sigma 2=1.602 \times 10^{7}$, and $\sigma 3=0.00509$ and varied values of $P_{r}$.

\begin{tabular}{lllllllr}
\hline & \multicolumn{3}{c}{ HAM results } & & \multicolumn{3}{c}{ Numerical results } \\
$P_{r}$ & $f^{\prime \prime}(0)$ & $-\theta^{\prime}(0)$ & $-H^{\prime}(0)$ & $-G^{\prime}(0)$ & $f^{\prime \prime}(0)$ & $-\theta^{\prime}(0)$ & $-H^{\prime}(0)$ \\
\hline 0.5 & 3.1059 & 1.2983 & -0.0996 & 0.0398 & 3.1059 & 1.2983 & -0.0996 \\
0.6 & 3.1059 & 1.3545 & -0.0996 & 0.0398 & 3.1059 & 1.3545 & -0.0996 \\
0.7 & 3.1059 & 1.4096 & -0.0996 & 0.0398 & 3.1059 & 1.4096 & -0.0996 \\
0.8 & 3.1059 & 1.4638 & -0.0996 & 0.0398 & 3.1059 & 1.4638 & -0.0996 \\
\hline
\end{tabular}


TABle 10: Computation for $f^{\prime \prime}(0),-\theta^{\prime}(0),-H^{\prime}(0)$, and $-G^{\prime}(0)$ with $P_{r}=0.5, S=-0.5, L=0.01, \delta=1, Q=0.01, R=0.1, S c=2.5, K 1=0.3$, $K 2=0.1, H a=0.2, \phi=0.02, \phi 1=\phi 2=0.01, \rho 1=5180, \rho 2=8900, \rho 3=1056, \sigma 1=0.74 \times 10^{6}, \sigma 2=1.602 \times 10^{7}$, and $\sigma 3=0.00509$ and varied values of $\delta$.

\begin{tabular}{cccccccc}
\hline & \multicolumn{3}{c}{ HAM results } & \multicolumn{3}{c}{ Numerical results } \\
$\delta$ & $f^{\prime \prime}(0)$ & $-\theta^{\prime}(0)$ & $-H^{\prime}(0)$ & $-G^{\prime}(0)$ & $f^{\prime \prime}(0)$ & $-\theta^{\prime}(0)$ & $-H^{\prime}(0)$ \\
\hline 1 & 3.1059 & 1.2983 & -0.0994 & 0.0994 & 3.1059 & 1.2983 & -0.0994 \\
2 & 3.1059 & 1.2983 & -0.0996 & 0.0398 & 3.1059 & 1.2983 & -0.0996 \\
3 & 3.1059 & 1.2983 & -0.0996 & 0.0332 & 3.1059 & 1.2983 & -0.0996 \\
4 & 3.1059 & 1.2983 & -0.0996 & 0.0249 & 3.1059 & 1.2983 & -0.0996 \\
\hline
\end{tabular}

TABle 11: Computation for $f^{\prime \prime}(0),-\theta^{\prime}(0),-H^{\prime}(0)$, and $-G^{\prime}(0)$ with $P_{r}=0.5, S=-0.5, L=0.01, \delta=1, R=0.1, S c=2.5, K 1=0.3$, $K 2=0.1, H a=0.2, \phi=0.02, \phi 1=\phi 2=0.01, \rho 1=5180, \rho 2=8900, \rho 3=1056, \sigma 1=0.74 \times 10^{6}, \sigma 2=1.602 \times 10^{7}$, and $\sigma 3=0.00509$ and varied values of $Q$.

\begin{tabular}{lllllllr}
\hline & \multicolumn{3}{c}{ HAM results } & \multicolumn{3}{c}{ Numerical results } \\
$Q$ & $f^{\prime \prime}(0)$ & $-\theta^{\prime}(0)$ & $-H^{\prime}(0)$ & $-G^{\prime}(0)$ & $f^{\prime \prime}(0)$ & $-\theta^{\prime}(0)$ & $-H^{\prime}(0)$ \\
\hline 0.01 & 3.1059 & 0.9457 & -0.0994 & 0.0994 & 3.1059 & 0.9457 & -0.0994 \\
0.02 & 3.1059 & 0.9465 & -0.0994 & 0.0994 & 3.1059 & 0.9465 & -0.0994 \\
0.03 & 3.1059 & 0.9472 & -0.0994 & 0.0994 & 3.1059 & 0.9472 & -0.0994 \\
0.04 & 3.1059 & 0.9480 & -0.0994 & 0.0994 & 3.1059 & 0.9480 & -0.0994 \\
\hline
\end{tabular}

TABle 12: Computation for $f^{\prime \prime}(0),-\theta^{\prime}(0),-H^{\prime}(0)$, and $-G^{\prime}(0)$ with $P_{r}=0.5, S=-0.5, L=0.01, \delta=1, Q=0.01, R=0.1, S c=2.5$, $K 1=0.3, K 2=0.1, H a=0.2, \phi=0.02, \phi 1=\phi 2=0.01, \rho 1=5180, \rho 2=8900, \rho 3=1056, \sigma 1=0.74 \times 10^{6}, \sigma 2=1.602 \times 10^{7}$, and $\sigma 3=$ 0.00509 and varied values of $R$.

\begin{tabular}{llllllll}
\hline & \multicolumn{3}{c}{ HAM results } & \multicolumn{3}{c}{ Numerical results } \\
$R$ & $f^{\prime \prime}(0)$ & $-\theta^{\prime}(0)$ & $-H^{\prime}(0)$ & $-G^{\prime}(0)$ & $f^{\prime \prime}(0)$ & $-\theta^{\prime}(0)$ & $-H^{\prime}(0)$ \\
\hline 0.1 & 3.1059 & 0.9457 & -0.0994 & 0.0994 & 3.1059 & 0.9457 & -0.0994 \\
0.2 & 3.1059 & 0.9515 & -0.0994 & 0.0994 & 3.1059 & 0.9515 & -0.0994 \\
0.3 & 3.1059 & 0.9562 & -0.0994 & 0.0994 & 3.1059 & 0.9562 & -0.0994 \\
0.4 & 3.1059 & 0.9601 & -0.0994 & 0.0994 & 3.1059 & 0.9601 & -0.0994 \\
\hline
\end{tabular}

velocity is increased for continued positive changes in $\mathrm{Ha}$ values while the same behavior is displayed as in the figure, for both situations. It is also found that the maximum velocity of HNF is greater than that of SNF. The impact on the temperature profile of the Prandtl number $P_{r}$ is seen in Figure 4. It is examined to show $\theta(\eta)$ in the case of SNF and the inclining performance for high values of $P_{r}$ in the case of HNF. Essentially, larger $P_{r}$ values enhance the boundary layer thickness that stimulates the nanoparticle's cooling effect due to thermal diffusion ratio. As contrast to in the HNF nanoparticles are tightly packed SNF. The impact on the temperature profile of $Q$ (heat generation parameter) is seen in Figure 4. For rising values of heat parameter $Q$, $T_{u}>T_{l}$ indicates a further transfer of heat from the surface into the fluid, thus raising the fluid temperature for both SNF and HNF. The growing temperature behavior in SNF and HNF for the larger value of $R$ is shown in Figure 4. However, it is commonly recognized that the heat transfer phenomena of their radiation process emit the energy via fluid particles, so that more heat is created during flow. Thus, the thermal boundary layer with more $R$ is defined as a development. Figure 5 indicates a rise in the Schmidt number $S c$ for SNF and HNF concentration profile of $H(\eta)$ and $G(\eta)$ and, as a result, reduction in the thickness of concentration boundary layer. The reactant concentration is observed to be increasing at a quicker rate when the diffusion coefficient of species is reduced; i.e., higher $S c$ values lead to a more rapid increase of the flow field concentration. The impact of homogeneous parameter $k 1$ and a heterogeneous parameter $k 2$ on the concentration profile $H(\eta)$ can be seen in Figure 6. Increase in the concentration profiles $H(\eta)$ it is observed that due to increase in $k 1$. According to this an increase in the homogeneous chemical reaction parameter which decreases viscosity. The concentration profile increases as the thickness of the boundary layer decreases for lower strength values of homogeneous reaction parameter. It is also proved that concentration profile is lower for the situation of HNF when compared with SNF. In both situations of SNF and HNF, the concentration boundary layer of the reactants decreases. However, they coincide for smaller values of $\eta$ which physically means that the homogeneous and heterogeneous reactions have no effect on the concentration of the reactants. The strong conduct of heterogeneous reaction parameters $K 2$ on the distribution is studied in Figure 6. The distribution of the concentration is increasing towards the surface of the plate and decreasing away from the surface with smaller values of $K 2$. When compared to HNF, the concentration distribution is smaller (SNF). It is noticed in Figure 6 that the behavior of concentration profile $G(\eta)$ and $H(\eta)$ is opposite. This shows the effects of the homogeneous chemical reaction parameter $k 1$ and the heterogeneous chemical reaction parameter $k 2$ on 

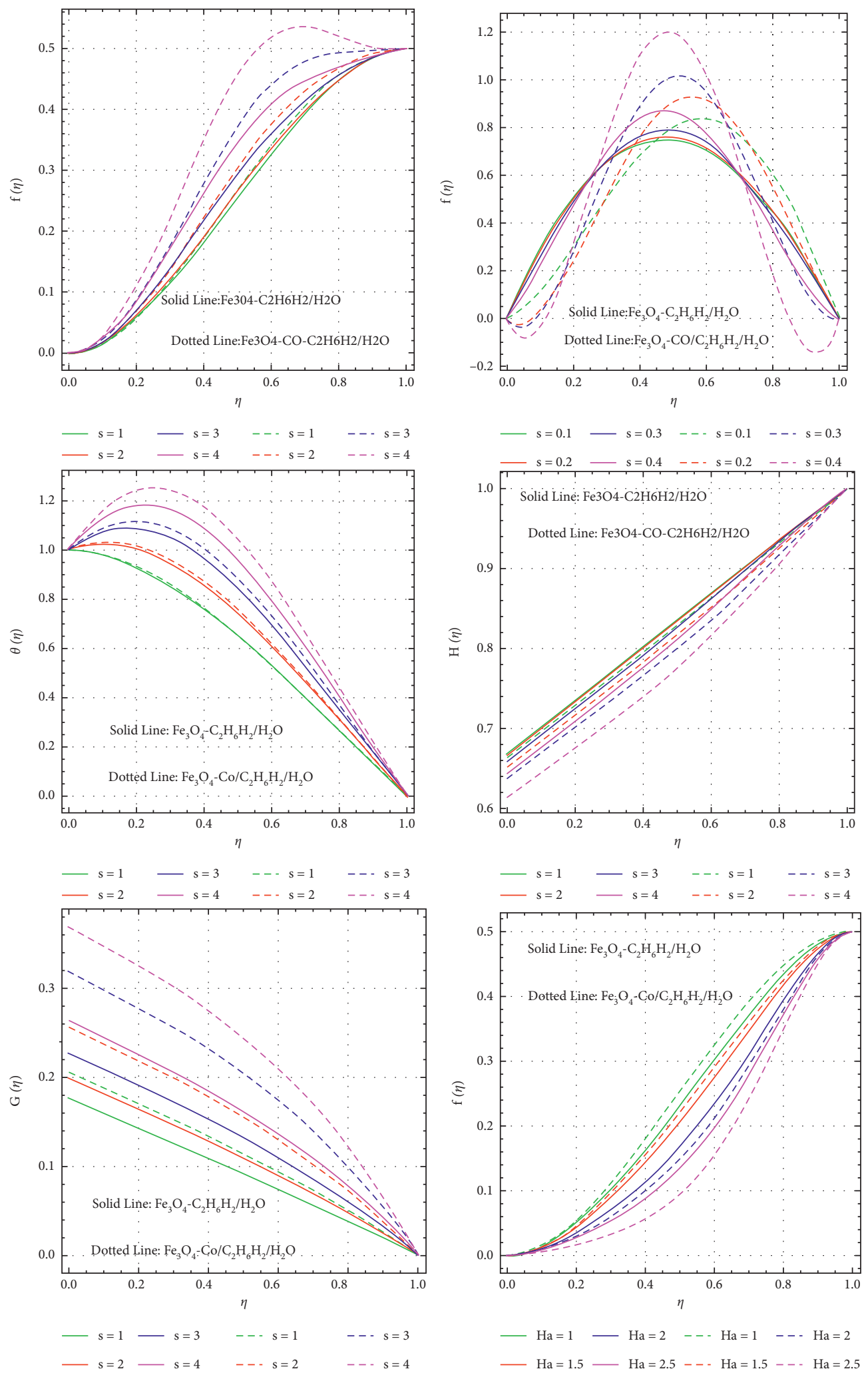

Figure 3: Impact of $S$ for $\mathrm{Fe}_{3} \mathrm{O}_{4}-\mathrm{C}_{2} \mathrm{H}_{6} \mathrm{O}_{2}-\mathrm{H}_{2} \mathrm{O}$ and $\mathrm{Fe}_{3} \mathrm{O}_{4}-\mathrm{Co} / \mathrm{C}_{2} \mathrm{H}_{6} \mathrm{O}_{2}-\mathrm{H}_{2} \mathrm{O}$ on $f(\eta), f^{\prime}(\eta), \theta(\eta), H(\eta), G(\eta)$ and impact of $H a$ on $f(\eta)$ with $L=2, \delta=1, Q=4, R=2, S c=P_{r}=H a=K 1=K 2=0.5, \phi=0.02, \phi 1=\phi 2=0.01, \rho 1=5180, \rho 2=8900, \rho 3=1056$, $\sigma 1=0.74 \times 10^{6}$, and $\sigma 2=1.602 \times 10^{7}, \sigma 3=0.00509$. 

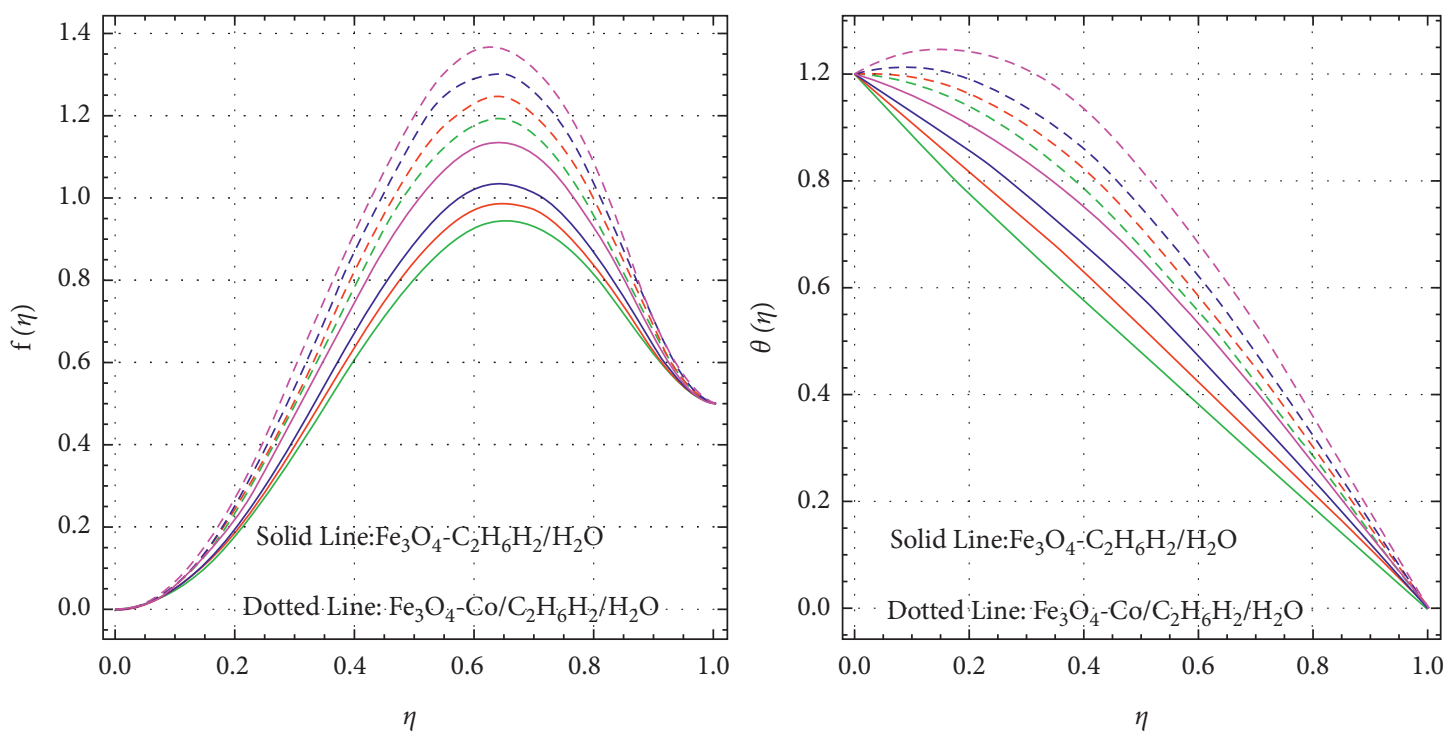

$\begin{array}{ll}-\mathrm{Q}=5 & --\mathrm{Q}=5 \\ -\mathrm{Q}=6 & --\mathrm{Q}=6 \\ -\mathrm{Q}=7 & --\mathrm{Q}=7 \\ \mathrm{Q}=8 & --\mathrm{Q}=8\end{array}$

\begin{tabular}{|c|c|}
\hline $\mathrm{Q}=5$ & $--\mathrm{Q}=5$ \\
\hline $\mathrm{Q}=5.5$ & $---Q=5.5$ \\
\hline$Q=6$ & $--Q=6$ \\
\hline $\mathrm{Q}=6.5$ & $-\mathrm{Q}=6.5$ \\
\hline
\end{tabular}
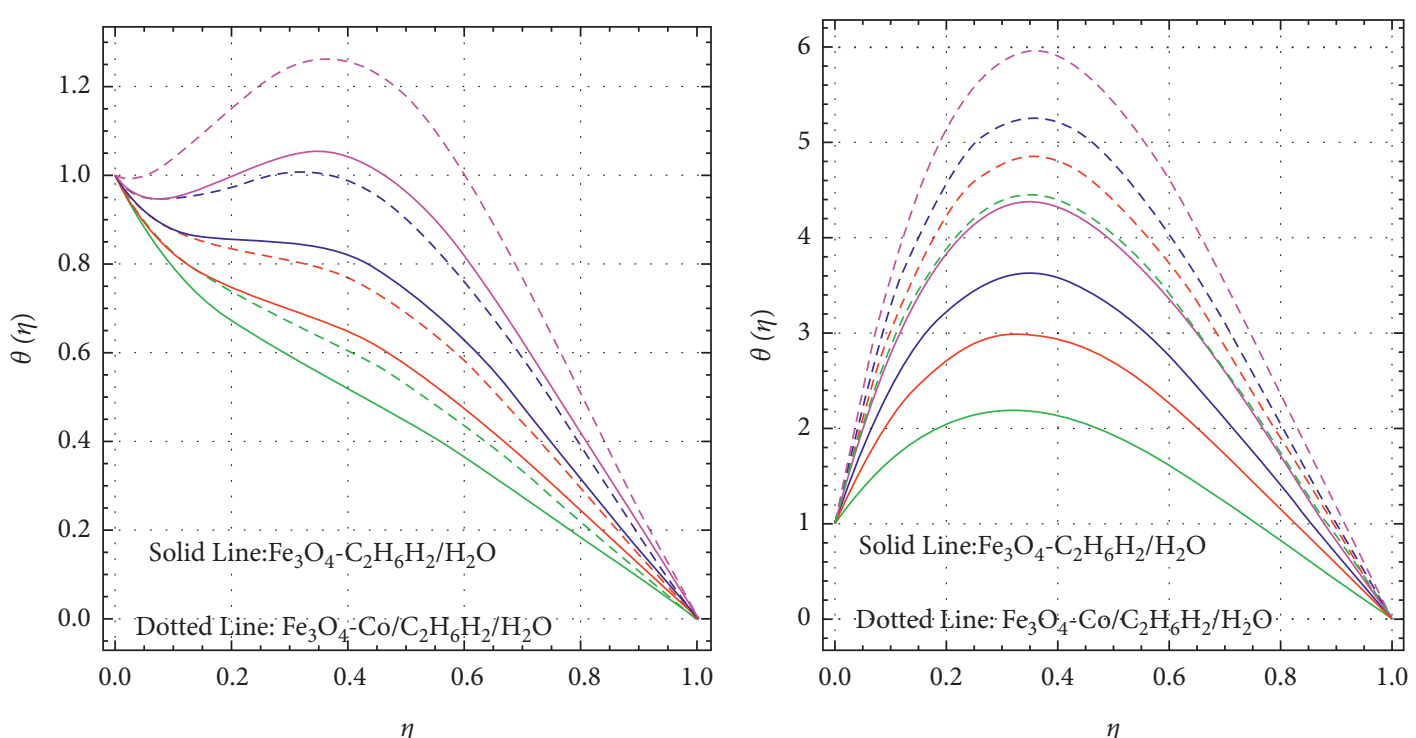

$\begin{aligned}-\operatorname{Pr}=5 & --\operatorname{Pr}=5 \\ -\operatorname{Pr}=6 & --\operatorname{Pr}=5 \\ -\operatorname{Pr}=7 & --\operatorname{Pr}=7 \\ -\operatorname{Pr}=8 & --\operatorname{Pr}=8\end{aligned}$

\begin{tabular}{|c|c|}
\hline $\mathrm{R}=5$ & $--\mathrm{R}=5$ \\
\hline $\mathrm{R}=5.5$ & $-R=5.5$ \\
\hline$R=6$ & $---R=6$ \\
\hline $\mathrm{R}=6.5$ & $-\quad R=6.5$ \\
\hline
\end{tabular}

Figure 4: Impact of $Q$ for $\mathrm{Fe}_{3} \mathrm{O}_{4}-\mathrm{C}_{2} \mathrm{H}_{6} \mathrm{O}_{2}-\mathrm{H}_{2} \mathrm{O}$ and $\mathrm{Fe}_{3} \mathrm{O}_{4}-\mathrm{Co} / \mathrm{C}_{2} \mathrm{H}_{6} \mathrm{O}_{2}-\mathrm{H}_{2} \mathrm{O}$ on $f(\eta)$ and $\theta(\eta)$ and impacts of $P_{r}$ and $R$ on $\theta(\eta)$ with $L=2, \delta=1, Q=4, R=2, S c=P_{r}=H a=K 1=K 2=0.5, \phi=0.02, \phi 1=\phi 2=0.01, \rho 1=5180, \rho 2=8900, \rho 3=1056, \sigma 1=0.74 \times 10^{6}$, $\sigma 2=1.602 \times 10^{7}$, and $\sigma 3=0.00509$.

the concentration profiles $H(\eta)$ and $G(\eta)$. It has been observed that increasing $k 1$ causes the concentration profiles $H(\eta)$ and $G(\eta)$ to increase. This is due to the fact that as the homogeneous chemical reaction parameter rises, the viscosity declines. However, the heterogeneous parameter $k 2$ yields the opposite result, as shown in Figure 6. This is because as $k 2$ increases, diffusion decreases, and the concentration of less diffused particles grows. The impacts of nanoparticles volume fraction $\phi$ on the velocity profiles $f(\eta), \theta(\eta), H(\eta)$, and $G(\eta)$ in case of SNF and HNF are shown in Figure 7. It can be clearly seen that the volume fraction parameter $\phi$ is increased with the increase in the 

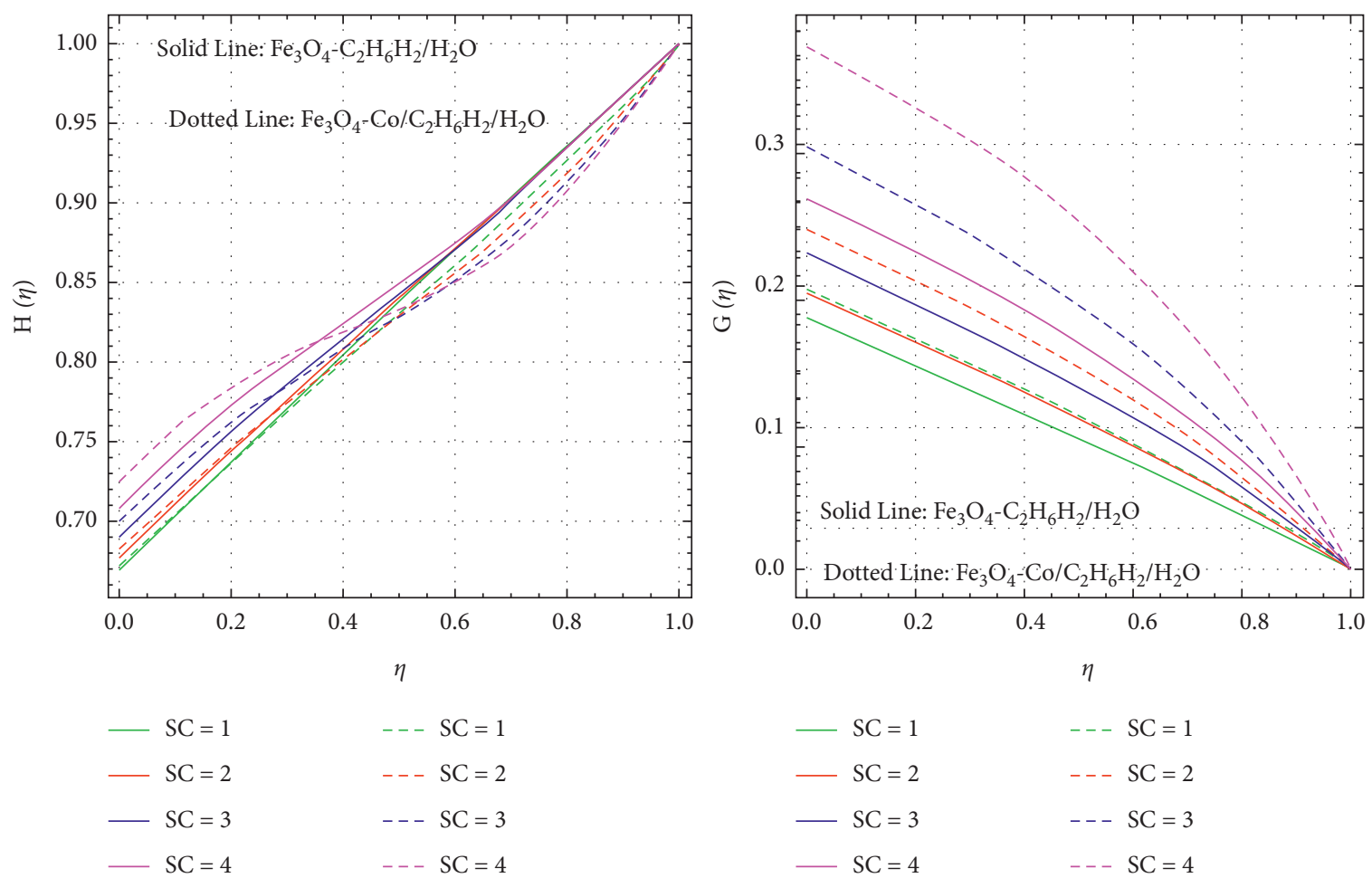

Figure 5: Impact of $S c$ for $\mathrm{Fe}_{3} \mathrm{O}_{4}-\mathrm{C}_{2} \mathrm{H}_{6} \mathrm{O}_{2}-\mathrm{H}_{2} \mathrm{O}$ and $\mathrm{Fe}_{3} \mathrm{O}_{4}-\mathrm{Co} / \mathrm{C}_{2} \mathrm{H}_{6} \mathrm{O}_{2}-\mathrm{H}_{2} \mathrm{O}$ on $H(\eta)$ and $G(\eta)$ with $L=2, \delta=1, Q=4, R=2$, $S=P_{r}=H a=K 1=K 2=0.5, \quad \phi=0.02, \quad \phi 1=\phi 2=0.01, \quad \rho 1=5180, \quad \rho 2=8900, \quad \rho 3=1056, \quad \sigma 1=0.74 \times 10^{6}, \quad \sigma 2=1.602 \times 10^{7}, \quad$ and $\sigma 3=0.00509$.

velocity for both SNF and HNF. In this physical problem the increase in velocity is because of the converse relationship that exists between the dynamic viscosity of nanofluid and the volume fraction. Consequently, the consistency of regular liquid is reduced against an increase in $\phi$, and therefore the fluid flow is supported up. The quantities related to the local coefficient of skin friction, the local Nusselt number, and the Sherwood number (homogeneous-heterogeneous reactions), that is, $f(\eta), \theta(\eta), H(\eta)$, and $G(\eta)$, for various values of $\phi$ are depicted in Figure 7. The values of $f(\eta)$ increase with increasing $\phi$, whereas the heat transfer rate $\theta(\eta)$ decreases. The values of homogeneous $H(\eta)$ increase with the increase in $\phi$, whereas the values of heterogeneous $G(\eta)$ decrease. It is also seen from these figures that the values of $f(\eta), \theta(\eta), H(\eta)$, and $G(\eta)$ are always positive. The influence on concentration profiles $f(\eta), \theta(\eta), H(\eta)$, and $G(\eta)$ of the parameter $S$ can be observed in Figure 8 in nanoparticles. All the concentration profiles are increased while $G(\eta)$ decreases, it is observed that due to increase in $S$. Figure 9 indicates that the $f(\eta)$ and $H(\eta)$ concentration profiles have increased with rising values of Schmidt number Sc, while $\theta(\eta)$ and $G(\eta)$ decrease. Figure 10 displays the effects of the homogeneous and heterogeneous chemical reaction parameters $k 1$ and $k 2$ on the concentration profiles $f(\eta), H(\eta)$, and $G(\eta)$. It has been observed that $k 1$ increases and $k 2$ decreases on the concentration profile $f(\eta)$; both decrease on the concentration profile $H(\eta)$; and both increase on $G(\eta)$. The purpose of Tables $5-12$ is to test the impact of various physical parameters numerically. As can be seen from the tables, all of the results are in good settlement with the BVP4c and HAM results. It is observed that effects of skin friction coefficient, velocity, temperature, and Nusselt and Sherwood numbers, both homogeneous as well as the heterogeneous parameters cause increment in the mass transfer rate. A decrease in the skin friction coefficient, as well as the heat and mass transfer rate, is caused by an increment in the internal heat generation parameter. A similar set of results has been observed when the squeezing parameter $S$ is increased. As the squeezing parameter $S$ is enhanced, the friction factor reduces and the local Nusselt and Sherwood numbers increase. The skin friction coefficient showed a downward trend, showing that the fluid was being drawn by the floor. Tables 3-12, respectively, show the results of $f(\eta), \theta(\eta), H(\eta), G(\eta), f^{\prime}(\eta),-\theta^{\prime}(\eta),-H^{\prime}(\eta)$, and $-G^{\prime}(\eta)$.

It is acknowledged from Table 5 that perhaps the skin friction factor $f^{\prime}(\eta)$ tends to increase the squeezing number $S$; the disruptive effect can be seen for the Hartmann number $\mathrm{Ha}$ and the volume fraction $\phi$ of nanoparticles and hybrid nanoparticles. From Table 6, it has been noticed that the squeezing number $S$ has declining influence on heat transfer efficiency, but Prandtl number $P_{r}, Q$ (heat generation parameter), radiation $R$, and volume fraction $\phi$ of nanoparticles and hybrid nanoparticles are greatly influencing the heat transfer efficiency. Table 7 depicts the behavior of nanoparticles and hybrid nanoparticles on $-H^{\prime}(\eta)$ and $-G^{\prime}(\eta)$ when $\delta=1$. It shows the decreasing values of $-H^{\prime}(\eta)$ and increasing values of squeezing number $S$, Schmidt number $S c$, and homogeneous reaction strength $K 1$, but values of 

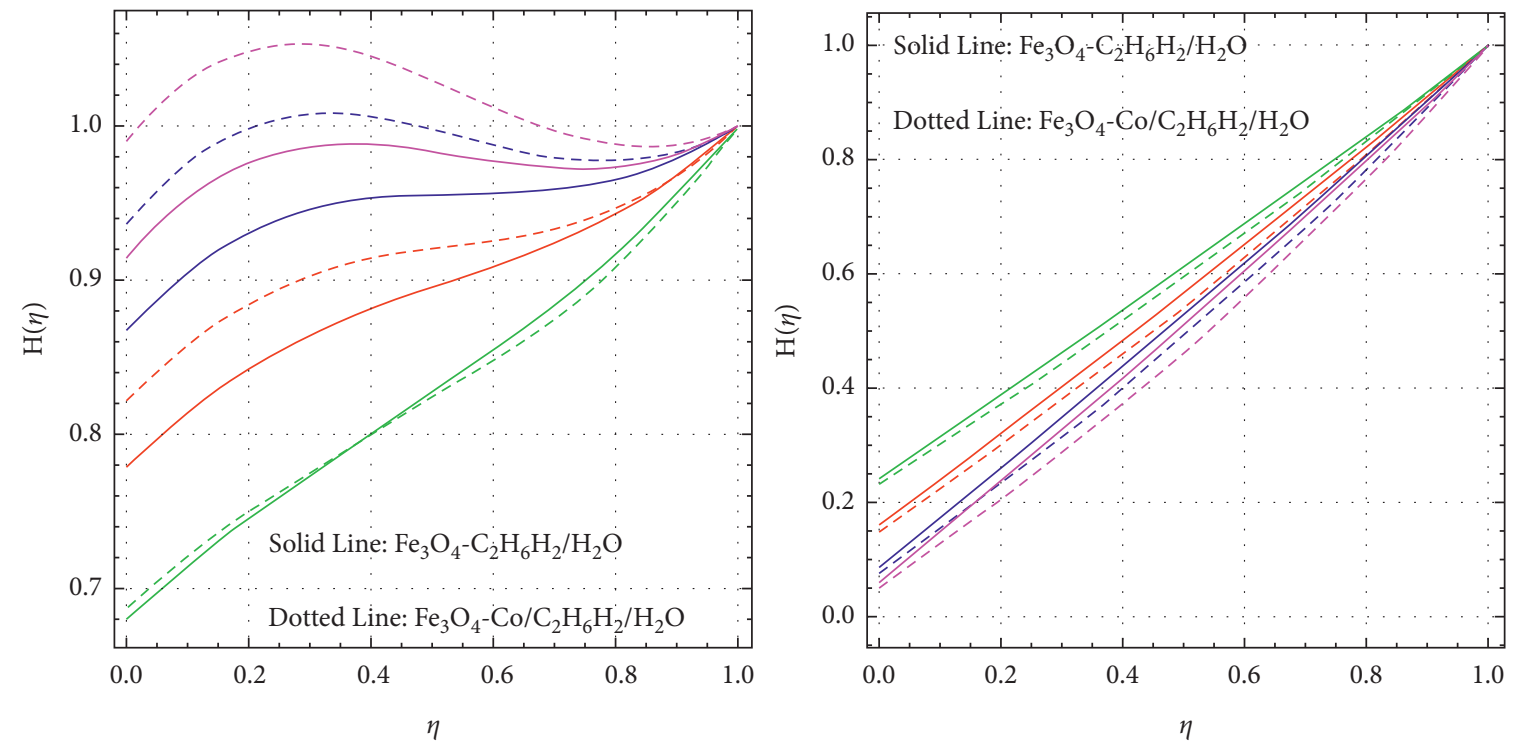

$$
\begin{aligned}
& -\mathrm{k} 1=1 \\
& \text { - - } \mathrm{k} 1=1 \\
& \text { - } \mathrm{k} 1=5 \\
& \text {-- } \mathrm{k} 1=5 \\
& -\mathrm{k} 1=10 \\
& \text { - - } \mathrm{k} 1=10 \\
& \text { - } \mathrm{k} 1=15 \\
& \text { - - } \mathrm{k} 1=15
\end{aligned}
$$
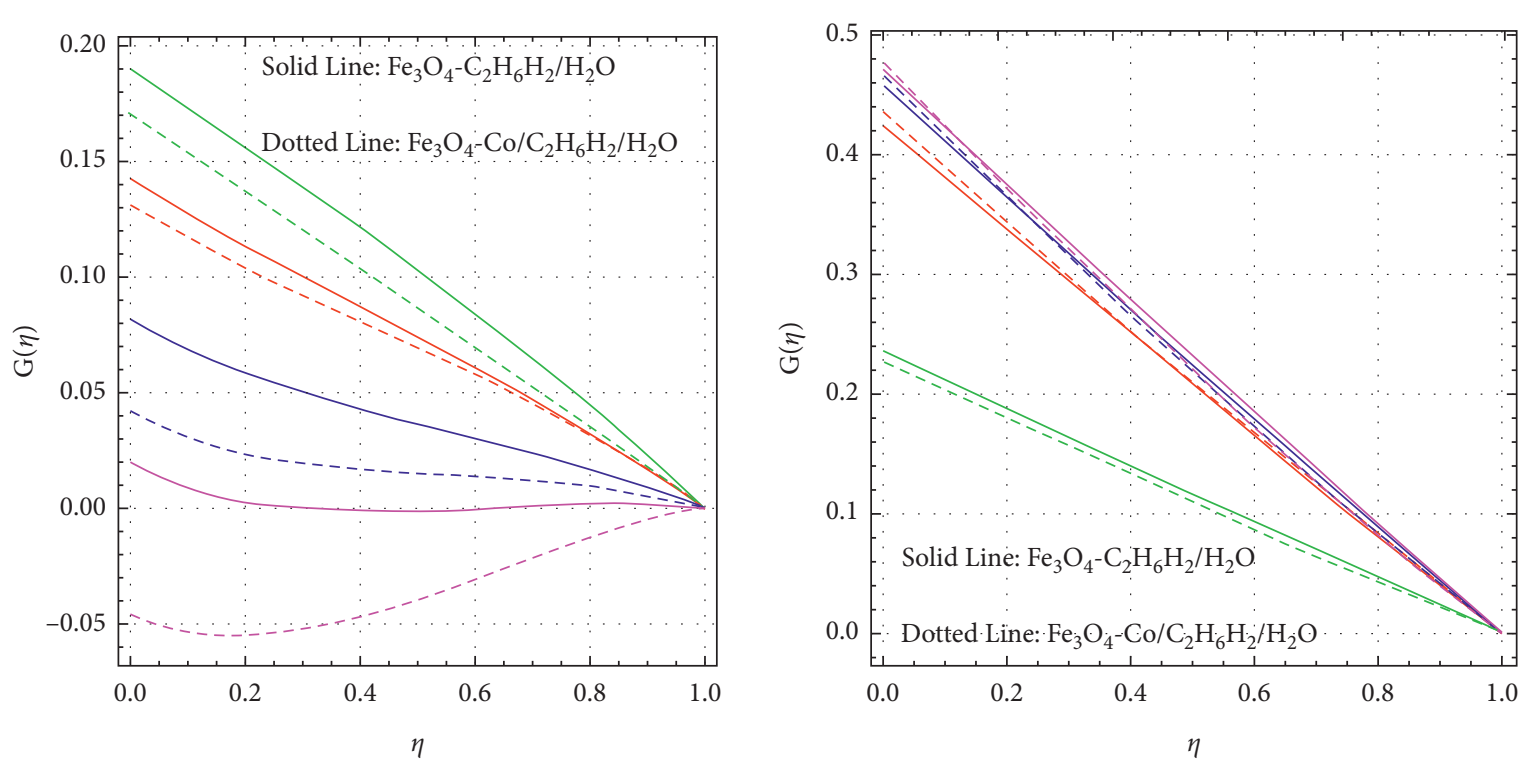

$$
\begin{aligned}
-\mathrm{k} 1 & =1 \\
-\mathrm{k} 1 & =5 \\
\mathrm{k} 1 & =10 \\
\mathrm{k} 1 & =15
\end{aligned}
$$$$
-\mathrm{k} 1=1
$$$$
---\mathrm{k} 1=1
$$$$
\text { - } \mathrm{k} 1=5
$$$$
\text { - - } \mathrm{k} 1=5
$$$$
-\mathrm{k} 1=10
$$$$
\text { - - } \mathrm{k} 1=10
$$

- $\mathrm{k} 1=15$

$$
---\mathrm{k} 1=15
$$

Figure 6: Impact of $K 1$ and $K 2$ for $\mathrm{Fe}_{3} \mathrm{O}_{4}-\mathrm{C}_{2} \mathrm{H}_{6} \mathrm{O}_{2}-\mathrm{H}_{2} \mathrm{O}$ and $\mathrm{Fe}_{3} \mathrm{O}_{4}-\mathrm{Co} / \mathrm{C}_{2} \mathrm{H}_{6} \mathrm{O}_{2}-\mathrm{H}_{2} \mathrm{O}$ on $H(\eta)$ and $G(\eta)$ with $L=2, \delta=1, Q=4$, $R=2, S c=P_{r}=H a=0.5, \phi=0.02, \phi 1=\phi 2=0.01, \rho 1=5180, \rho 2=8900, \rho 3=1056, \sigma 1=0.74 \times 10^{6}, \sigma 2=1.602 \times 10^{7}$, and $\sigma 3=0.00509$. 

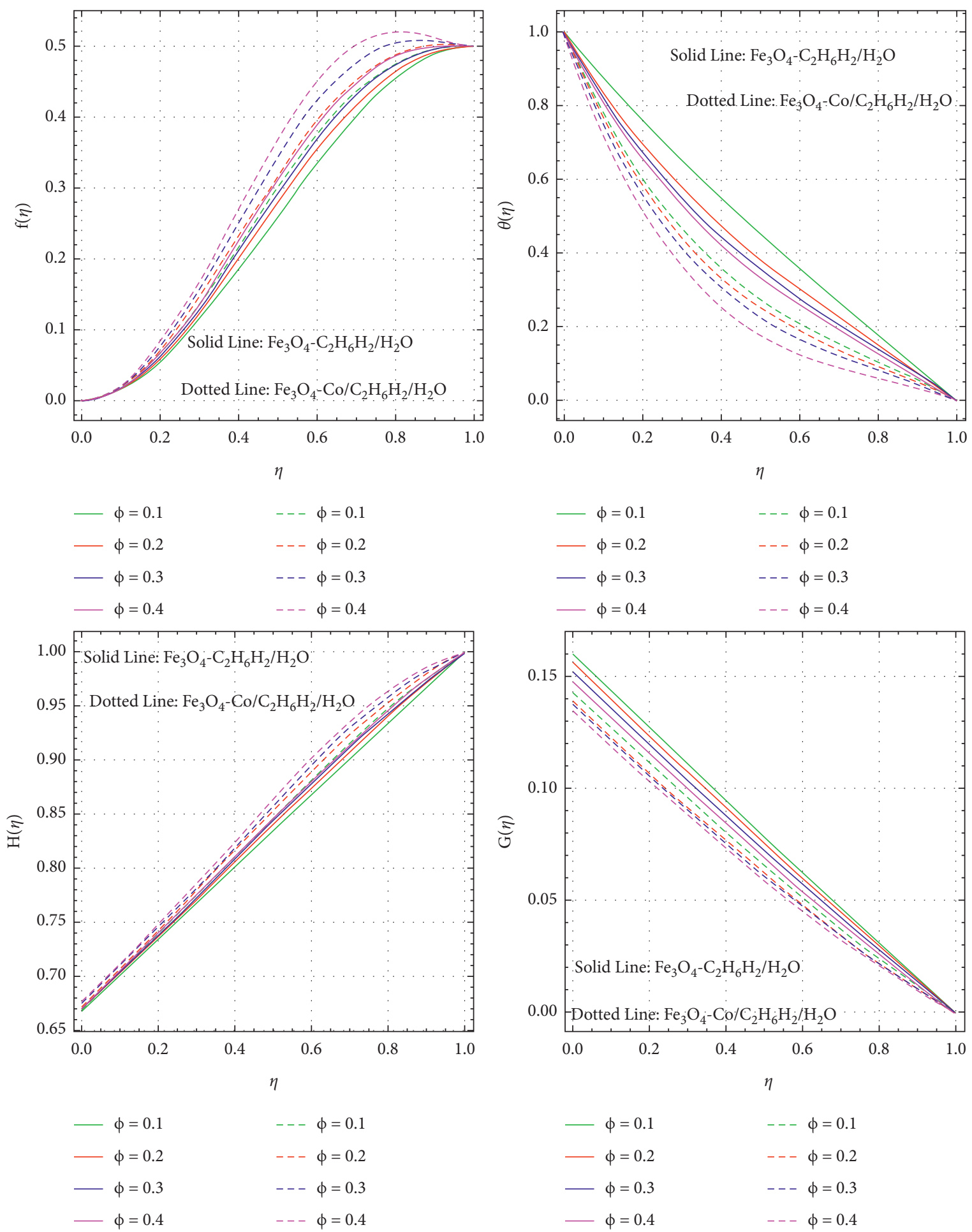

$$
\begin{aligned}
& -\phi=0.1 \\
& --\phi=0.1 \\
& \text { - } \phi=0.2 \\
& \text { - - } \phi=0.2 \\
& \phi=0.3 \\
& \text { - - } \phi=0.3 \\
& \phi=0.4 \\
& \text { - - } \phi=0.4
\end{aligned}
$$

Figure 7: Impact of $\phi$ for $\mathrm{Fe}_{3} \mathrm{O}_{4}-\mathrm{C}_{2} \mathrm{H}_{6} \mathrm{O}_{2}-\mathrm{H}_{2} \mathrm{O}$ and $\mathrm{Fe}_{3} \mathrm{O}_{4}-\mathrm{Co} / \mathrm{C}_{2} \mathrm{H}_{6} \mathrm{O}_{2}-\mathrm{H}_{2} \mathrm{O}$ on $f(\eta), \theta(\eta), H(\eta)$, and $G(\eta)$ with $L=2, \delta=1, Q=4$, $R=2, S c=P_{r}=H a=K 1=K 2=0.5, \phi=0.02, \phi 1=\phi 2=0.01, \rho 1=5180, \rho 2=8900, \rho 3=1056, \sigma 1=0.74 \times 10^{6}, \sigma 2=1.602 \times 10^{7}$, and $\sigma 3=0.00509$. 

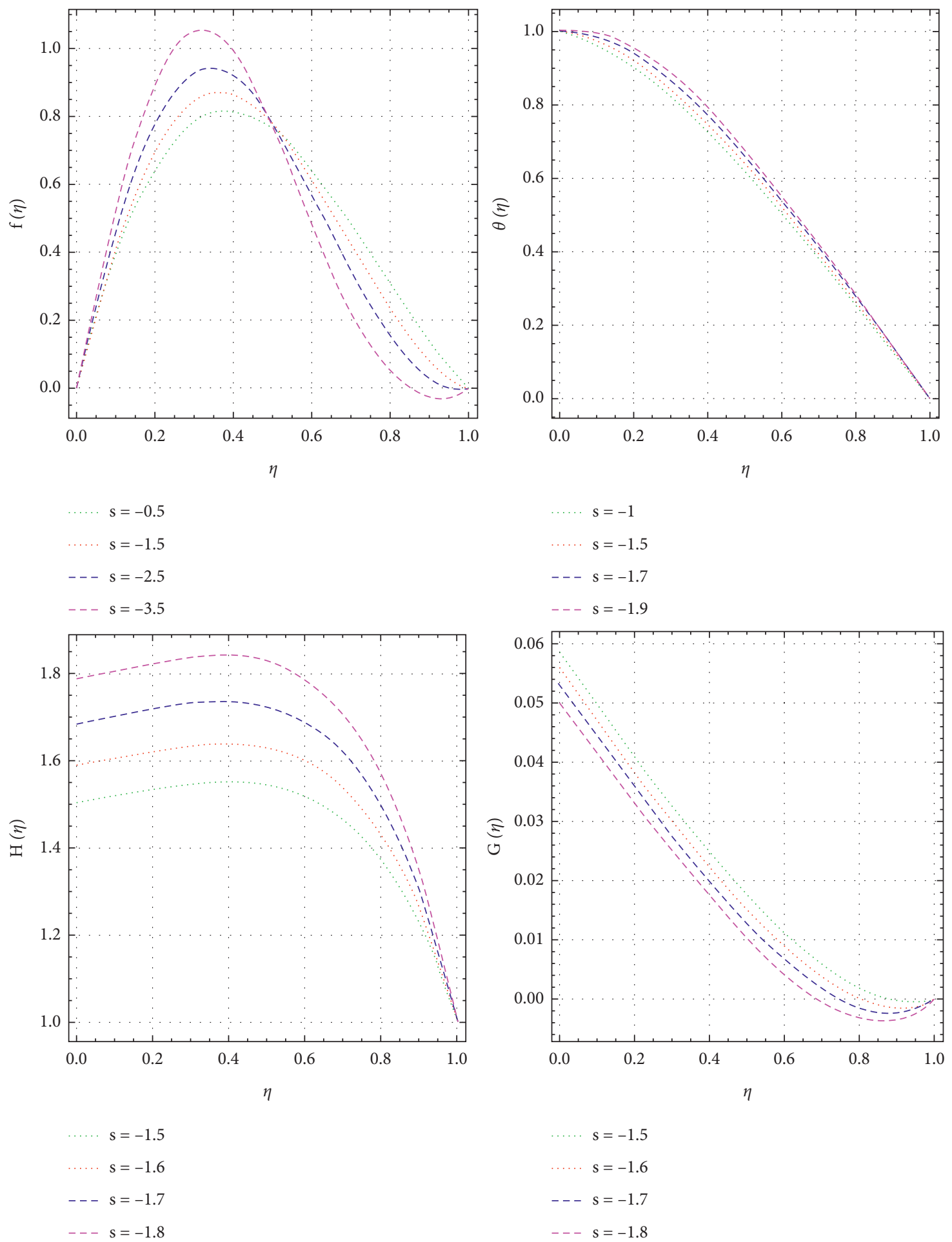

Figure 8: Impact of $S$ on $f(\eta), \theta(\eta), H(\eta)$, and $G(\eta)$ with $P_{r}=0.5, S=-0.5, L=0.01, \delta=1, Q=0.01, R=0.1, S c=2.5, K 1=0.3$, $K 2=0.1, H a=0.2, \phi=0.02, \phi 1=\phi 2=0.01, \rho 1=5180, \rho 2=8900, \rho 3=1056, \sigma 1=0.74 \times 10^{6}, \sigma 2=1.602 \times 10^{7}$, and $\sigma 3=0.00509$. 

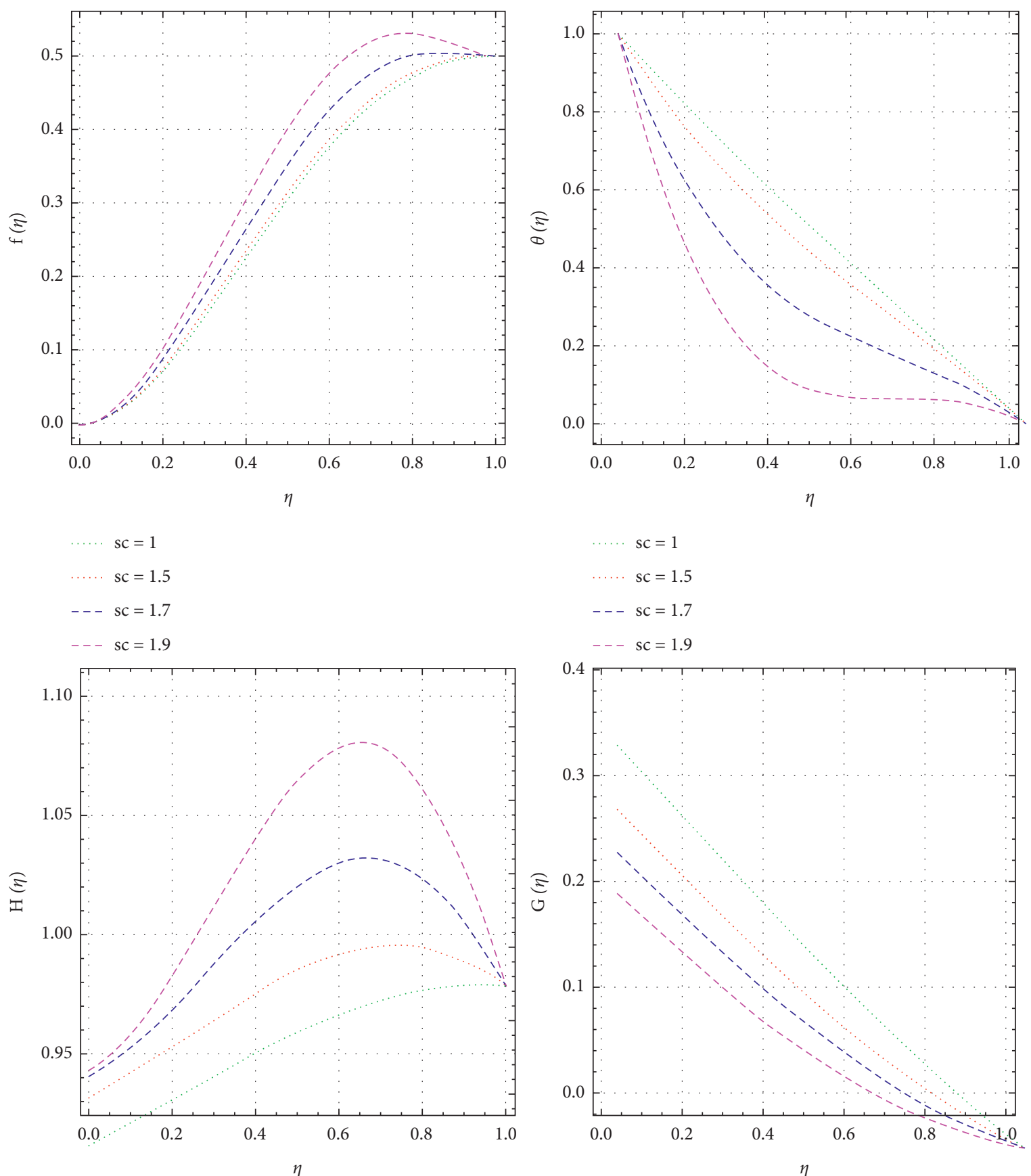

$$
\begin{aligned}
& \ldots . \cdot \mathrm{sc}=1 \\
& \ldots . \mathrm{sc}=1.5 \\
& ---\mathrm{sc}=1.7 \\
& --\mathrm{sc}=1.9
\end{aligned}
$$

$$
\begin{aligned}
& \ldots . . \mathrm{sc}=1 \\
& \ldots . \mathrm{sc}=1.5 \\
& ---\mathrm{sc}=1.7 \\
& --\mathrm{sc}=1.9
\end{aligned}
$$

Figure 9: Impact of $S c$ on $f(\eta), \theta(\eta), H(\eta)$, and $G(\eta)$ with $P_{r}=0.5, S=-0.5, L=0.01, R=1, H a=0.1, S c=2.5, K 1=0.3, K 2=0.1, \delta=1$, $\phi=0.02, \phi 1=\phi 2=0.01, \rho 1=5180, \rho 2=8900, \rho 3=1056, \sigma 1=0.74 \times 10^{6}, \sigma 2=1.602 \times 10^{7}$, and $\sigma 3=0.00509$. 

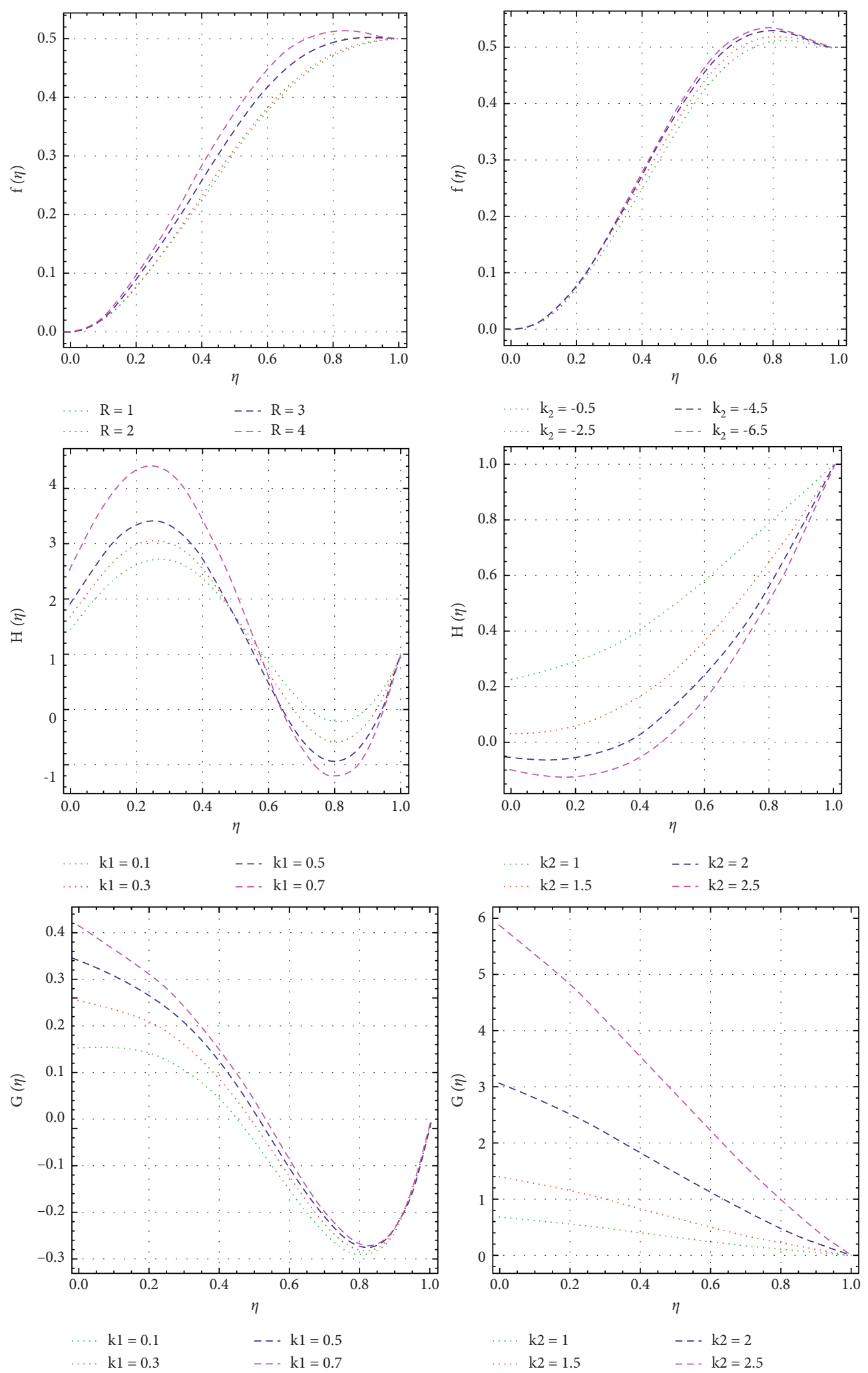

Figure 10: Impact of $K 1$ and $K 2$ on $f(\eta), H(\eta)$, and $G(\eta)$ with $P_{r}=1, S=-5, L=0.5, Q=5, R=1, \delta=1, S c=2.5, K 1=5, K 2=5$, $H a=0.5, \phi=0.02, \phi 1=\phi 2=0.01, \rho 1=5180, \rho 2=8900, \rho 3=1056, \sigma 1=0.74 \times 10^{6}, \sigma 2=1.602 \times 10^{7}$, and $\sigma 3=0.00509$. 
TABle 13: Computation for $f^{\prime \prime}(0),-\theta^{\prime}(0),-H^{\prime}(0)$, and $-G^{\prime}(0)$ with $P_{r}=0.5, S=-0.5, L=0.01, \delta=1, Q=0.01, R=0.1, S c=2.5, K 1=0.3$, $K 2=0.1, H a=0.2, \phi=0.02, \phi 1=\phi 2=0.01, \rho 1=5180, \rho 2=8900, \rho 3=1056, \sigma 1=0.74 \times 10^{6}, \sigma 2=1.602 \times 10^{7}$, and $\sigma 3=0.00509$ and varied values of $S c$.

\begin{tabular}{llllllll}
\hline & \multicolumn{3}{c}{ HAM results } & \multicolumn{3}{c}{ Numerical results } \\
Sc & $f^{\prime \prime}(0)$ & $-\theta^{\prime}(0)$ & $-H^{\prime}(0)$ & $-G^{\prime}(0)$ & $f^{\prime \prime}(0)$ & $-\theta^{\prime}(0)$ & $-H^{\prime}(0)$ \\
\hline 2.5 & 3.1059 & 0.9457 & -0.0994 & 0.0994 & 3.1059 & 0.9457 & -0.0994 \\
3.5 & 3.1059 & 0.9457 & -0.1044 & 0.1044 & 3.1059 & 0.9457 & -0.1044 \\
4.5 & 3.1059 & 0.9457 & -0.1106 & 0.1106 & 3.1059 & 0.9457 & -0.1106 \\
5.5 & 3.1059 & 0.9457 & -0.1200 & 0.1200 & 3.1059 & 0.9457 & -0.1200 \\
\hline
\end{tabular}

TABle 14: Computation for $f^{\prime \prime}(0),-\theta^{\prime}(0),-H^{\prime}(0)$, and $-G^{\prime}(0)$ with $P_{r}=0.5, S=-10, L=0.01, \delta=1, Q=0.01, R=0.1, S c=2.5$, $K 2=0.1, H a=0.2, \phi=0.02, \phi 1=\phi 2=0.01, \rho 1=5180, \rho 2=8900, \rho 3=1056, \sigma 1=0.74 \times 10^{6}, \sigma 2=1.602 \times 10^{7}$, and $\sigma 3=0.00509$ and varied values of $K 1$.

\begin{tabular}{llllllll}
\hline & \multicolumn{3}{c}{ HAM results } & \multicolumn{3}{c}{ Numerical results } \\
$K 1$ & $f^{\prime \prime}(0)$ & $-\theta^{\prime}(0)$ & $-H^{\prime}(0)$ & $-G^{\prime}(0)$ & $f^{\prime \prime}(0)$ & $-\theta^{\prime}(0)$ & $-H^{\prime}(0)$ \\
\hline 1.1 & 3.1059 & 0.9457 & -0.0994 & 0.0994 & 3.1059 & 0.9457 & -0.0994 \\
1.5 & 3.1059 & 0.9457 & -0.0995 & 0.0995 & 3.1059 & 0.9457 & -0.0995 \\
1.9 & 3.1059 & 0.9457 & -0.0997 & 0.0997 & 3.1059 & 0.9457 & -0.0997 \\
2.5 & 3.1059 & 0.9457 & -0.0999 & 0.0999 & 3.1059 & 0.9457 & -0.0999 \\
\hline
\end{tabular}

TABle 15: Computation for $f^{\prime \prime}(0),-\theta^{\prime}(0),-H^{\prime}(0)$, and $-G^{\prime}(0)$ with $P_{r}=0.5, S=-10, L=0.01, \delta=1, Q=0.01, R=0.1, S c=2.5$, $K 1=0.3, H a=0.2, \phi=0.02, \phi 1=\phi 2=0.01, \rho 1=5180, \rho 2=8900, \rho 3=1056, \sigma 1=0.74 \times 10^{6}, \sigma 2=1.602 \times 10^{7}$, and $\sigma 3=0.00509$ and varied values of $K 2$.

\begin{tabular}{|c|c|c|c|c|c|c|c|c|}
\hline \multicolumn{5}{|c|}{ HAM results } & \multicolumn{4}{|c|}{ Numerical results } \\
\hline$K 2$ & $f^{\prime \prime}(0)$ & $-\theta^{\prime}(0)$ & $-H^{\prime}(0)$ & $-G^{\prime}(0)$ & $f^{\prime \prime}(0)$ & $-\theta^{\prime}(0)$ & $-H^{\prime}(0)$ & $-G^{\prime}(0)$ \\
\hline 0.01 & 3.1059 & 0.9457 & -0.0994 & 0.0994 & 3.1059 & 0.9457 & -0.0994 & 0.0994 \\
\hline 0.02 & 3.1059 & 0.9457 & -0.1758 & 0.1758 & 3.1059 & 0.9457 & -0.1758 & 0.1758 \\
\hline 0.03 & 3.1059 & 0.9457 & -0.2362 & 0.2362 & 3.1059 & 0.9457 & -0.2362 & 0.2362 \\
\hline 0.04 & 3.1059 & 0.9457 & -0.2850 & 0.2850 & 3.1059 & 0.9457 & -0.2850 & 0.2850 \\
\hline
\end{tabular}

heterogeneous reaction strength $k 2$, for both $-H^{\prime}(\eta)$ and $-G^{\prime}(\eta)$, show incremental behavior. The volume fraction $\phi$ of nanoparticles and hybrid nanoparticles has the same values' behavior.

\section{Conclusion}

This investigation gives a mathematical answer for dissecting the impacts of flow between two squeezing plates with a homogeneous and heterogeneous reaction in the presence of hybrid nanoparticles. The impact of dimensional overseeing boundaries on velocity, temperature, profiles with skin friction, and local Nusselt and Sherwood numbers is examined with the assistance of graphs and tables. The effects of the current examination are listed below:

(i) The skin friction coefficient augmenting for growing values of solid volume fraction $\phi$.

(ii) The increasing values of the squeezing parameter $S$ reduced the friction factor and local Nusselt numbers, but from Table 7 Sherwood numbers are decreasing and increasing on the $-H(0)$ and $-G(0)$.

(iii) The Prandtl number, heat generation, and radiation parameters appear to increase in the local Nusselt numbers, as shown in Tables 9, 11, and 12. (iv) Homogeneous-heterogeneous parameters assist in observing the flow's utility profiles.

(v) The local Nusselt and friction factor in fluid viscosity are similar as $S c$ increases and the homogeneous parameter decreases while heterogeneous parameter increases, as shown in Table 13.

(vi) Tables 14 and 15 show that homogeneous reaction strength and heterogeneous reaction strength are decreasing and increasing on the profile of $-H(0)$ and $-G(0)$.

\section{Nomenclature}

$\begin{array}{ll}T: & \text { Temperature } \\ T_{u}: & \text { Upper plate's temperature } \\ T_{l}: & \text { Lower plate's temperature } \\ P: & \text { Pressure } \\ \rho_{h n f}: & \text { Effective density } \\ \left(\rho c_{p}\right)_{h n f}: & \text { Effective heat capacity } \\ \sigma_{h n f}: & \text { Electrical conductivity of nanofluid } \\ u, v: & \text { Nanofluid velocity } \\ D_{A}, D_{B}: & \text { Diffusion coefficients of the chemical species } \\ \delta: & \text { Ratio of the diffusion coefficients } \\ K^{*}: & \text { Permeability } \\ k_{h n f}: & \text { Nanofluid thermal conductivity }\end{array}$


$K_{1}$ : Homogeneous reaction strength

$K_{2}$ : Heterogeneous reaction strength

$S: \quad$ Squeeze number

$P_{r}: \quad$ Prandtl number

$\mathrm{Ha}$ : Hartmann number

$R: \quad$ Radiation parameter

Q: $\quad$ Heat generation parameter

L: $\quad$ Length

$C_{f}$ : Skin friction coefficient

Nu: $\quad$ Local Nusselt number

Sh: Local Sherwood number

N1, N2: Chemical species.

\section{Data Availability}

No data were used in this study.

\section{Conflicts of Interest}

The authors declare that there are no conflicts of interest.

\section{Acknowledgments}

The authors extend their appreciation to the Deanship of Scientific Research at King Khalid University, Saudi Arabia, for funding this work through research groups program under grant R.G.P.2/11/43.

\section{References}

[1] J. Stefan, "Versuchuber die scheinbare adhesion,Sitzunqsber Sachs kadwissein," Math-Nat Wiss Kl, vol. 69, pp. 713-721, 1874.

[2] T. Hayat, M. Nawaz, A. A. Hendi, and S. Asghar, "MHD squeezing flow of a micropolar fluid between parallel disks," Journal of Fluids Engineering, vol. 133, pp. 111206-111210, 2011.

[3] M. Mustafa, T. Hayat, and S. Obaidat, "On heat and mass transfer in the unsteady squeezing flow between parallel plates," Meccanica, vol. 47, no. 7, pp. 1581-1589, 2012.

[4] B. Mahanthesh, B. J. Gireesha, N. S. Shashikumar, T. Hayat, and A. Alsaedi, "Marangoni convection in Casson liquid flow due to an infinite disk with exponential space dependent heat source and cross-diffusion effects," Results in Physics, vol. 9, pp. 78-85, 2018.

[5] J. L. Neuringer, "Some viscous flows of a saturated ferrofluid under the combined influence of thermal and magnetic field gradients," International Journal of Non-Linear Mechanics, vol. 1, pp. 123-137, 1996.

[6] W. A. Khan, Z. H. Khan, and R. U. Haq, "Flow and heat transfer of ferrofluids over a flat plate with uniform heat flux," The European Physical Journal Plus, vol. 130, no. 4, Article ID 86, 2015.

[7] A. M. Rashad, "Impact of thermal radiation on MHD slip flow of a ferrofluid over a non-isothermal wedge," Journal of Magnetism and Magnetic Materials, vol. 422, pp. 25-31, 2017.

[8] A. Zaib, U. Khan, Z. Shah, P. Kumam, and P. Thounthong, "Optimization of entropy generation in flow of micropolar mixed convective magnetite (Fe3O4) ferroparticle over a vertical plate," Alexandria Engineering Journal, vol. 58, no. 4, pp. 1461-1470, 2019.

[9] L. Ali, X. Liu, B. Ali, S. Mujeed, S. Abdal, and S. A. Khan, "Analysis of magnetic properties of nano-particles due to a magnetic dipole in micropolar fluid flow over a stretching sheet," Coatings, vol. 10, no. 2, Article ID 170, 2020.

[10] S. Suresh, K. P. Venkitaraj, P. Selvakumar, and M. Chandrasekar, "Effect of $\mathrm{Al}_{2} \mathrm{O}_{3}-\mathrm{Cu}$ /water hybrid nanofluid in heat transfer," Experimental Thermal and Fluid Science, vol. 38, pp. 54-60, 2012.

[11] D. Madhesh and S. Kalaiselvam, "Experimental study on heat transfer and rheological characteristics of hybrid nanofluids for cooling applications," Journal of Experimental Nanoscience, vol. 10, no. 15, pp. 1194-1213, 2015.

[12] A. A. Minea, "Hybrid nanofluids based on $\mathrm{Al}_{2} \mathrm{O}_{3}, \mathrm{TiO}_{2}$ and $\mathrm{SiO}_{2}$ : numerical evaluation of different approaches," International Journal of Heat and Mass Transfer, vol. 104, pp. 852-860, 2017.

[13] T. Hayat and S. Nadeem, "Heat transfer enhancement with Ag-CuO/water hybrid nanofluid," Results in Physics, vol. 7, pp. 2317-2324, 2017.

[14] F. Mebarek-Oudina, "Convective heat transfer of Titania nanofluids of different base fluids in cylindrical annulus with discrete heat source," Heat Transfer-Asian Research, vol. 48, no. 1, pp. 135-147, 2019.

[15] B. Mahanthesh, B. J. Gireesha, I. L. Animasaun, T. Muhammad, and N. S. Shashikumar, "MHD flow of SWCNT and MWCNT nanoliquids past a rotating stretchable disk with thermal and exponential space dependent heat source," Physica Scripta, vol. 94, no. 8, Article ID 85214, 2019.

[16] S. Marzougui, F. Mebarek-Oudina, A. Assia, M. Magherbi, Z. Shah, and K. Ramesh, "Entropy generation on magnetoconvective flow of copper-water nanofluid in a cavity with chamfers," Journal of Thermal Analysis and Calorimetry, vol. 143, no. 3, pp. 2203-2214, 2020.

[17] A. Wakif, A. Chamkha, T. Thumma, I. L. Animasaun, and R. Sehaqui, "Thermal radiation and surface roughness effects on the thermo-magneto-hydrodynamic stability of aluminacopper oxide hybrid nanofluids utilizing the generalized Buongiorno's nanofluid model," Journal of Thermal Analysis and Calorimetry, vol. 143, no. 2, pp. 1201-1220, 2020.

[18] A. S. Alshomrani and T. Gul, "A convective study of $\mathrm{Al}_{2} \mathrm{O}_{3}$ $\mathrm{H}_{2} \mathrm{O}$ and $\mathrm{Cu}-\mathrm{H}_{2} \mathrm{O}$ nano-liquid films sprayed over a stretching cylinder with viscous dissipation," The European Physical Journal Plus, vol. 132, no. 11, Article ID 495, 2017.

[19] N. Sandeep and A. Malvandi, "Enhanced heat transfer in liquid thin film flow of non-Newtonian nanofluids embedded with graphene nanoparticles," Advanced Powder Technology, vol. 27, no. 6, pp. 2448-2456, 2016.

[20] N. Sandeep, "Effect of aligned magnetic field on liquid thin film flow of magnetic-nanofluids embedded with graphene nanoparticles," Advanced Powder Technology, vol. 28, no. 3, pp. 865-875, 2017.

[21] A. Ahmed and S. Nadeem, "Effects of magnetohydrodynamics and hybrid nanoparticles on a micropolar fluid with 6-types of stenosis," Results in Physics, vol. 7, pp. 4130-4139, 2017.

[22] S. Hamrelaine, F. Mebarek-Oudina, and M. R. Sari, "Analysis of MHD Jeffery Hamel flow with suction/injection by homotopy analysis method," Journal of Advanced Research in Fluid Mechanics and Thermal Sciences, vol. 58, pp. 173-186, 2019.

[23] K. Anantha Kumar, N. Sandeep, V. Sugunamma, and I. L. Animasaun, "Effect of irregular heat source/sink on the radiative thin film flow of MHD hybrid ferrofluid," Journal of Thermal Analysis and Calorimetry, vol. 139, no. 3, pp. 21452153, 2020.

[24] A. Zaib, U. Khan, A. Wakif, and M. Zaydan, "Numerical entropic analysis of mixed MHD convective flows from a non- 
isothermal vertical flat plate for radiative tangent hyperbolic blood biofluids conveying magnetite ferroparticles: dual similarity solutions," Arabian Journal for Science and Engineering, vol. 45, no. 7, pp. 5311-5330, 2020.

[25] A. Wakif, M. Qasim, M. I. Afridi, S. Saleem, and M. M. AlQarni, "Numerical examination of the entropic energy harvesting in a magnetohydrodynamic dissipative flow of Stokes' second problem: utilization of the gear-generalized differential quadrature method," Journal of Non-equilibrium Thermodynamics, vol. 44, no. 4, pp. 385-403, 2019.

[26] P. K. Kameswaran, S. Shaw, P. Sibanda, and P. V. S. N. Murthy, "Homogeneous-heterogeneous reactions in a nanofluid flow due to a porous stretching sheet," International Journal of Heat and Mass Transfer, vol. 57, no. 2, pp. 465-472, 2013.

[27] M. A. Chaudhary and J. H. Merkin, "Free-convection stagnation-point boundary layers driven by catalytic surface reactions: I the steady states," Journal of Engineering Mathematics, vol. 28, no. 2, pp. 145-171, 1994.

[28] R. Jawad and M. R. Azizah, "Numerical investigation of copper-water (Cu-water) nanofluid with different shapes of nanoparticles in a channel with stretching wall: slip effects," Mathematical and Computational Applications, vol. 21, pp. 43-58, 2016.

[29] J. H. Merkin, "A model for isothermal homogeneous-heterogeneous reactions in boundary-layer flow," Mathematical and Computer Modelling, vol. 24, no. 8, pp. 125-136, 1996.

[30] M. Sheikholeslami, M. M. Rashidi, D. M. Al Saad, F. Firouzi, B. RokiniH, and G. Domairrry, "Steady nanofluid flow between parallel plates considering thermophoresis and Brownian effects," Journal of King Saud University Science, vol. 49, 2015. 